\title{
Effect of inhomogeneities on streamer propagation: I. Intersection with isolated bubbles and particles
}

\author{
Natalia Yu Babaeva and Mark J Kushner ${ }^{1}$ \\ Department of Electrical Engineering and Computer Science, University of Michigan, 1301 Beal Ave., \\ Ann Arbor, MI 48109, USA \\ E-mail:nbabaeva@umich.edu and mjkush@umich.edu
}

Received 26 December 2008, in final form 8 April 2009

Published 29 May 2009

Online at stacks.iop.org/PSST/18/035009

\begin{abstract}
The branching of streamers in high pressure gas discharges and discharges in liquids is an almost universal occurrence having many causes. In this paper, we discuss results of an investigation of one possible cause-inhomogeneities in the media through which the streamer propagates. These inhomogeneities produce corresponding enhancements or decreases in ionization and excitation as the avalanche front encounters them, some of which may produce branching. Three types of inhomogeneities were investigated-negative bubbles (regions having a lower density than ambient), positive bubbles (having a higher density) and solid bubbles (particles). Depending on the size and density of the bubble, the streamer can be focused into the bubble (negative small bubble), deflected and split (positive bubbles and particles) or refracted (large negative bubble). In the case of gaseous bubbles, this behavior is partly explained by the larger $E / N$ (electric field/gas number density) in the negative bubble, producing more ionization by electron avalanche, and smaller $E / N$ in the positive bubble, producing less ionization. A streamer may diverge into a negative bubble located off axis due to seeding of electrons in the bubble by photoionization and subsequent avalanching in the large $E / N$.
\end{abstract}

(Some figures in this article are in colour only in the electronic version)

\section{Introduction}

Streamer branching is a common phenomenon that occurs in most high pressure gases and liquids [1-4]. The investigation of branching is of interest from both scientific and technological perspectives. For example, the branching of short pulse corona discharges in part determine the uniformity of forming radicals during plasma initiated combustion [5] and in plasma remediation of toxic gases [6]. By branching we refer to the splitting of a discharge filament or streamer into two or more streamers. A typical scenario is the following. The high $E / N$ (electric field/gas number density) avalanche front of a positive streamer trails a conductive, low $E / N$ channel a few hundred micrometers in diameter. The streamer comes to a point where, what appears to be spontaneously, the streamer splits into two (or more) avalanche fronts, each

\footnotetext{
${ }^{1}$ Author to whom any correspondence should be addressed.
}

trailing its own channel. A streamer can branch many times-a single streamer launches daughter streamers which themselves launch daughter streamers. Some of the streamers may terminate or stall prior to bridging the anode-cathode gap.

Branching has been attributed to many causes, such as fluctuations in the size of the avalanche front or photoionization initiated by UV photons generated in the avalanche front [1]. Branching in streamers depends on gas composition, electrode geometry and on polarity and magnitude of the applied voltage. In general, higher voltages tend to produce more streamers and more streamers that survive to bridge the gap between electrodes [7]. The evolution of the streamer may depend on its local environment and the proximity of other streamers. A mutual electro-dynamical influence exerted from one streamer on another has been observed [8]. The dependence of discharge branching on pressure is complex. In general, branching increases with increasing pressure. At lower pressures in air ( $<380$ Torr) streamers tend to propagate with single 
diffusive channels. As the pressure is increased to 500 Torr, the streamers begins to branch. Approaching atmospheric pressure, simultaneous production of new branches may occur [9]. The streamer radius is generally inversely proportional to pressure $[9,10]$.

It has also been observed that inhomogeneities in the path or near the path of a streamer may initiate branching. These inhomogeneities locally enhance or diminish ionizationsuch as polarized solid particles [11], water droplets [12,13], surfaces with enhanced secondary electron emission [14], or regions of high temperature or low density that increases the local $E / N$ [15]. For example, it has been found that liquid droplets in the path of streamers sustained in air may both guide the direction and alter the speed of the streamer [11]. Oladiran investigated the interaction of positive streamers at atmospheric pressure with charged and uncharged water droplets, and found branching correlated with the droplets $[12,13]$.

There are also observations that streamer channels not only branch and repel each other, but may merge or reconnect $[16,17]$. Luque et al [18] suggested that two competing mechanisms may affect streamer interactions-electrostatic repulsion and attraction through non-local photoionization. A recent study using stereo-photography [19] suggest that visually merging streamers may cross behind each other.

Few computational investigations have addressed branching phenomena or interactions between multiple streamers. For example, Naidis addressed the interaction between simultaneously propagating streamers in atmospheric pressure air [20]. Numerical studies by Ebert and co-workers have shown that a Laplacian instability can occur at the leading edge of a streamer that produces spontaneous branching at the tip [21-24]. Recent analytical theory addressing this instability has agreed well with the computations. Another possible component to branching is the stochastic processes occurring at or in front of the leading edge of the avalanche front that randomly places seed electrons ahead of the steamer [25].

The consequences of streamers encountering inhomogeneities in their paths are important from many technological applications. For example, high voltage initiated streamers in fuel-air mixtures are being investigated for producing radicals to increase the flame speed and decrease the detonation time $[5,26]$. These mixtures are typically highly inhomogeneous and may contain both fuel vapor and fuel aerosols or droplets. Streamers intersecting with solid particles is the desired outcome in electrostatic precipitators [27]. Inhomogeneities can also be encountered by streamers in liquids. A liquid can contain solid particles (for example, as a result of electrode erosion) or gaseous bubbles. These inhomogeneities can be the source of enhanced ionization and play an important role in streamer propagation through an otherwise dense media.

It is likely that under non-pristine conditions, some component of streamer branching and instabilities can be attributed to inhomogeneities or fluctuations of density, pressure or temperature in or near the streamer path. The two extremes are gas bubbles in liquids and particles in gases. Computational investigations have shown that the interaction of streamers with solid particles can be complex and depend on the conductivity and dielectric constant of the particle [28].

To lend insights into the role of inhomogeneities in streamer propagation, in this paper, we discuss results from a two-dimensional computational investigation of the intersection of a positive corona streamer sustained in humid atmospheric pressure air with inhomogeneities in the background gas in or near its path. These inhomogeneities are referred to as bubbles. Three varieties of bubbles were investigated, negative bubbles (gas region having a lower density than ambient), positive bubbles (having a higher density) and solid bubbles or particles. We found that the intersection of a streamer with a bubble can significantly alter its path and properties, and, under certain conditions, produce branching. The size and position of the bubble plays an important role in possible branching. For example, a streamer accelerates and penetrates into a negative bubble located on the streamer axis but does not branch. A streamer may, however, diverge off axis into a negative bubble located off axis. A streamer encountering a positive bubble on axis will likely branch around it whereas a streamer decelerates and avoids a positive bubble when it is located off axis. These interactions depend on the manner of seeding electrons ahead of the streamer through photoionization. The pressure difference between the bubbles and the ambient gas investigated here were restricted to be $<7-8 \%$. The consequences of highly rarefied fields of bubbles in multi-atmospheric pressure air are discussed in Part II [29].

The use of a clearly defined negative bubble with sharp boundaries in a gas is not necessarily intended to map onto a common, naturally occurring structure. Low density regions in gases would at best be transient with a smooth transition between dense and rarefied regions. One could artificially create negative bubbles by, for example, focusing a pulsed infrared laser in air. Rather our negative bubbles are intended to be somewhat artificial structures to provide a well-defined system to illustrate the properties of streamers propagating through regions of varied density, as might occur for streamers in liquids with bubbles.

The model used in this study is described in section 2. Typical streamer characteristics in the absence of inhomogeneities are discussed in section 3. Consequences of streamers interacting with positive and negative gas bubbles, and solid bubbles located on axis are discussed in sections 4 and 5. Section 6 addresses streamer dynamics with bubbles of different sizes and locations. The role of the mean free path of ionizing photons is discussed in section 7. Concluding remarks are in section 8 .

\section{Description of the model}

The physical processes and algorithms used in the model are discussed in detail in [28] and so will be only briefly reviewed here. The model, nonPDPSIM, is a multi-fluid hydrodynamics simulation in which transport equations for all charged and neutral species and Poisson's equation are integrated as a function of time. The fundamental equations for charged 
species that are solved are

$$
\begin{gathered}
-\nabla \cdot \varepsilon \nabla \Phi=\sum_{j} N_{j} q_{j}+\rho_{\mathrm{s}}, \\
\frac{\partial N_{j}}{\partial t}=-\nabla \cdot \vec{\phi}_{j}+S_{j}, \\
\frac{\partial \rho_{\mathrm{s}}}{\partial t}=\sum_{j} q_{j}\left(-\nabla \cdot \vec{\phi}_{j}+S_{j}\right)-\nabla \cdot(\sigma(-\nabla \Phi)),
\end{gathered}
$$

where $\varepsilon, \Phi, \rho_{\mathrm{s}}$ and $\sigma$ are the permittivity, electric potential, surface charge and conductivity of solid materials; and for species $j, N_{j}, \phi_{j}, S_{j}$ and $q_{j}$ are density, flux, source function and charge. Poisson's equation (equation (1)), transport equations for conservation of the charged species $j$ (equation (2)) and the material and surface charge balance equation (equation (3)) are simultaneously integrated using a sparse-matrix and Newton iteration technique.

Updates of the charged particle densities and electric potential are followed by an implicit update of the electron temperature by solving the electron energy conservation equation

$\frac{\partial \varepsilon}{\partial t}\left(n_{\mathrm{e}} \varepsilon\right)=\vec{j} \cdot \vec{E}-\nabla \cdot\left(-\kappa \nabla T_{\mathrm{e}}+\frac{5}{2} \varepsilon \vec{\phi}_{\mathrm{e}}\right)-n_{\mathrm{e}} \sum_{i} \Delta \varepsilon_{i} k_{i} N_{i}$,

where $n_{\mathrm{e}}$ is the electron density, the average electron energy $\varepsilon=\frac{3}{2} k T_{\mathrm{e}}$ for electron temperature $T_{\mathrm{e}}, \vec{\phi}_{\mathrm{e}}$ is the ScharfetterGummel form of the electron flux, $\vec{j}=q \vec{\phi}_{\mathrm{e}}$ is the total electron current in electric field $\vec{E}$ and $\kappa$ is the electron thermal conductivity. The summation is over electron collisions with species having density $N_{i}$ and rate coefficient $k_{i}$ resulting in change in electron energy $\Delta \varepsilon_{i}$. The electron transport coefficients and rate coefficients for bulk electrons as a function of $T_{\mathrm{e}}$ are obtained by solving Boltzmann's equation for the electron energy distribution (EED). (Although the nonMaxwellian nature of the EED is captured in our solving Boltzmann's equation, we will refer to $T_{\mathrm{e}}=(2 / 3) \varepsilon$, where $\varepsilon$ is the average electron energy.) As the streamer propagation occurs on times of less than tens of nanoseconds, we did not address the bulk, pressure driven hydrodynamic motion of the neutral gas though we did include diffusive transport of neutrals.

Poisson's equation (equation (1)) was solved throughout the entire computational domain (except in metals where the potential is specified as a boundary condition). Continuity equations for gas phase charged and neutral particles are only solved in the plasma region. Equation (3) for surface and volume charges is solved on and inside all non-metallic materials.

Photoionization by streamer generated radiation producing electrons ahead of the avalanche front is critical to the propagation of positive streamers [30-34]. Our approach to photoionization is based on line-of-sight propagation of UV ionizing radiation generated by high lying excited states that are produced largely in the high $E / N$ in the avalanche front. The UV radiation is absorbed (without producing ionization) over a specified mean free path which determines its extent beyond its origin. Photoionization occurs by absorption of UV radiation by selected species. The source term in equation (2) includes these photoionization processes which are computed using Green's functions. The rate of photoionization is given by

$$
\begin{gathered}
\frac{\partial N_{i}^{+}(\vec{r})}{\partial t}=N_{\mathrm{i}}(\vec{r}) \sum_{j} \int N_{j}\left(\vec{r}^{\prime}\right) A_{j} G_{j}\left(\vec{r}, \vec{r}^{\prime}\right) \sigma_{i j} \mathrm{~d}^{3} \vec{r}^{\prime}, \\
G_{j}\left(\vec{r}, \vec{r}^{\prime}\right)=\frac{\exp \left(-\sum_{k} \int_{\vec{r}^{\prime}}^{\vec{r}} N_{k}\left(\vec{r}^{\prime \prime}\right) \sigma_{k j} \mathrm{~d} \vec{r}^{\prime \prime}\right)}{4 \pi\left|\vec{r}-\vec{r}^{\prime}\right|^{2}} .
\end{gathered}
$$

In these expressions, excited state $N_{j}$ emits a photon at location $\vec{r}^{\prime}$ with a rate given by the Einstein coefficient $A_{j}$ and ionizes species $N_{i}$ with cross section $\sigma_{i j}$ at location $\vec{r}$. In traversing the plasma the photons are absorbed by species $N_{k}$ with cross section $\sigma_{k j}$. The function $G_{j}\left(\vec{r}, \vec{r}^{\prime}\right)$ is the probability of survival of the emitted photon and divergence of its flux between emission and ionization. We assumed that ionization occurs by absorption by $\mathrm{O}_{2}$ of photons emitted by $\mathrm{N}_{2}\left(b^{1} \Pi\right)$ and $\mathrm{N}_{2}\left(b^{1} \Sigma\right)$ in the wavelength range $98-102 \mathrm{~nm}$. The non-ionizing absorption cross section was approximated as $2 \times 10^{-17} \mathrm{~cm}^{-2}$ while that for the ionizing cross section as $10^{-18} \mathrm{~cm}^{-2}$. In the absence of inhomogeneities, the precise value of these cross sections does not significantly affect the calculation other than in the speed of propagation of the streamer provided that the ionizing cross section is large enough to prevent the streamer from stalling. With inhomogeneities, the likelihood of branching is sensitive to both the absorption length and photoionization cross section, as both determine the seeding of electrons in and near a bubble [29]. Although it is true that the photoionization mechanism and the detailed trends we discuss below are particular to humid air, the trends are fairly general. Calculations performed for similar conditions in argon with both a different photoionization mechanism and lack of electronegative component show similar trends.

The gas mixture is atmospheric pressure humid air $\mathrm{N}_{2} / \mathrm{O}_{2} / \mathrm{H}_{2} \mathrm{O}=79.5 / 19.5 / 1$ at $300 \mathrm{~K}$. The species included are $\mathrm{N}_{2}, \mathrm{~N}_{2}(\mathrm{v}), \mathrm{N}_{2} *, \mathrm{~N}_{2} * *, \mathrm{~N}_{2}^{+}, \mathrm{N}, \mathrm{N}^{*}, \mathrm{~N}^{+}, \mathrm{N}_{4}^{+}, \mathrm{O}_{2}, \mathrm{O}_{2} *, \mathrm{O}_{2}^{+}$, $\mathrm{O}_{2}^{-}, \mathrm{O}^{-}, \mathrm{O}, \mathrm{O}^{*}, \mathrm{O}^{+}, \mathrm{O}_{3}, \mathrm{H}_{2} \mathrm{O}, \mathrm{H}_{2} \mathrm{O}^{+}, \mathrm{H}_{2}, \mathrm{H}, \mathrm{OH}$ and electrons. The states $\mathrm{N}^{*}$ and $\mathrm{N}^{* *}$ are nominally $\mathrm{N}_{2}\left(A^{3} \Sigma\right)$ and $\mathrm{N}_{2}\left(b^{1} \Pi\right.$, $\left.b^{1} \Sigma\right)$ though the latter is treated as a lumped state including transitions higher than $\mathrm{N}_{2}\left(A^{3} \Sigma\right)$. $\mathrm{N}^{*}$ is nominally $\mathrm{N}\left({ }^{2} \mathrm{D}\right)$ but is also intended to be a lumped state for higher excitation as well. The states $\mathrm{O}^{*}$ and $\mathrm{O}_{2} *$ are nominally $\mathrm{O}\left({ }^{1} \mathrm{D}\right)$ and $\mathrm{O}_{2}\left({ }^{1} \Delta\right)$ but are also intended to be lumped states. To initiate the streamer, a small spot of seed-charges (electrons and $\mathrm{N}_{2}^{+}$with peak density of $10^{8} \mathrm{~cm}^{-3}$, diameter of $250 \mu \mathrm{m}$ ) was placed $10 \mu \mathrm{m}$ from the tip of the anode. The precise magnitude and diameter of the seed plasma had little effect on the results other than the speed with which the streamer is initiated. There was otherwise no initial plasma density elsewhere in the computational domain.

The model geometry is shown in figure 1 . The positive corona discharge is sustained between a rod encased in a dielectric $\left(\varepsilon / \varepsilon_{0}=5\right)$ having an exposed edge with a radius of curvature of $0.07 \mathrm{~cm}$. The electrode is biased to $15 \mathrm{kV}$ and is separated from a flat grounded electrode by $2 \mathrm{~mm}$. The unstructured numerical mesh has triangular elements with 

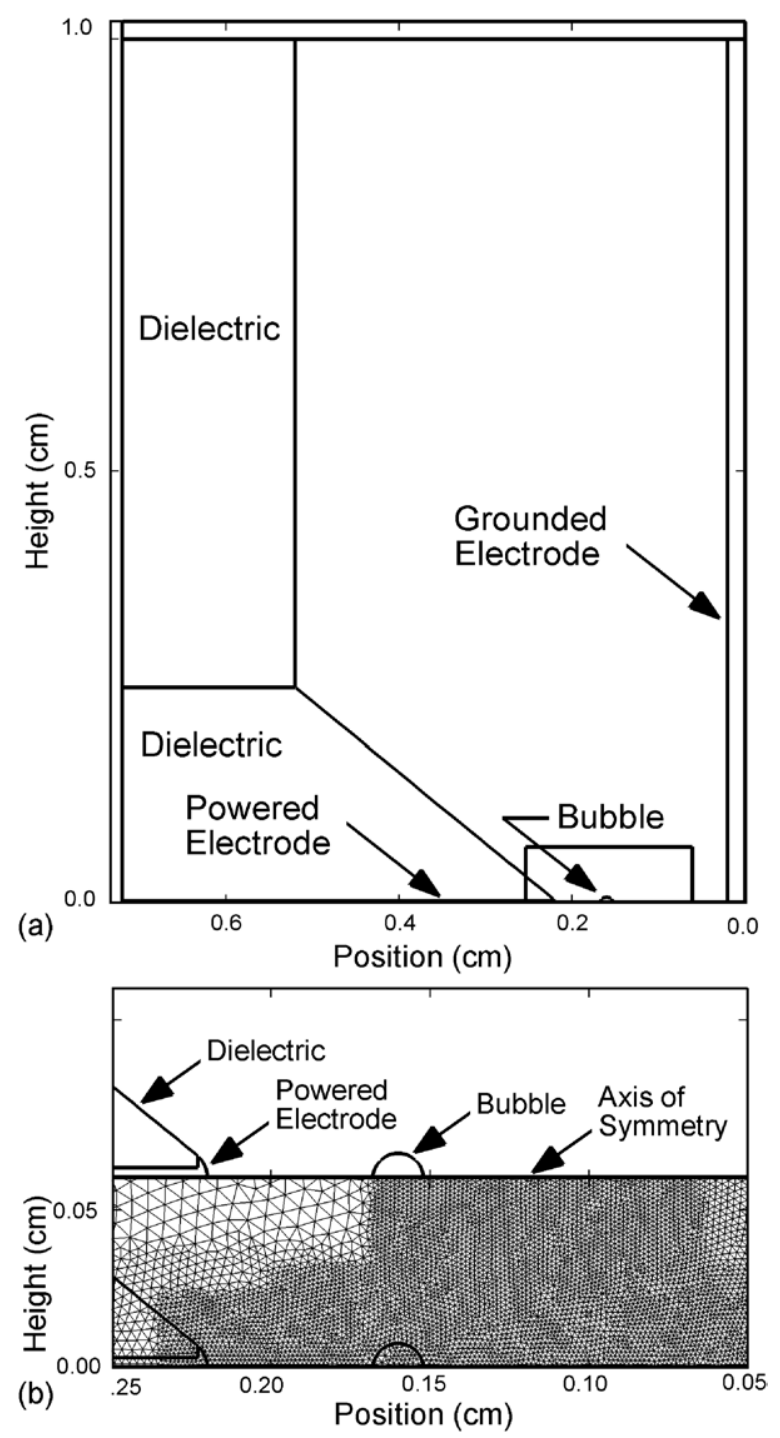

Figure 1. Schematic of the corona discharge. (a) Entire computational domain and $(b)$ close up of the anode-cathode gap with typical numerical meshing for one bubble.

refinement regions to resolve the detail of the electrode tip and the bubble as well as the larger surrounding features. The schematic shown in figure $1(b)$ has a bubble of $80 \mu \mathrm{m}$ radius placed on axis. The mesh consisted of approximately 5000-7000 nodes, of which about 4000-6000 are in the plasma region. The mesh resolution is $1-4 \mu \mathrm{m}$ near the anode and bubbles. The results discussed here address streamer propagation in the vicinity of a single bubble with small pressure perturbations and with dimensions comparable to that of the streamer $(\leqslant 320 \mu \mathrm{m})$.

The issues studied here are, as is the case for many streamer phenomena, three-dimensional by nature. Our 2D axially symmetric, Cartesian simulation is an approximation whereby the bubbles actually appear to be rods. The weakness of this approximation is two fold. First, the streamer is less likely to intersect with a spherical bubble of a given radius in a three-dimensional space. Second, streamers launched by a bubble, discussed in more detail in Part II, are less likely to intersect with other streamers launched by other bubbles.

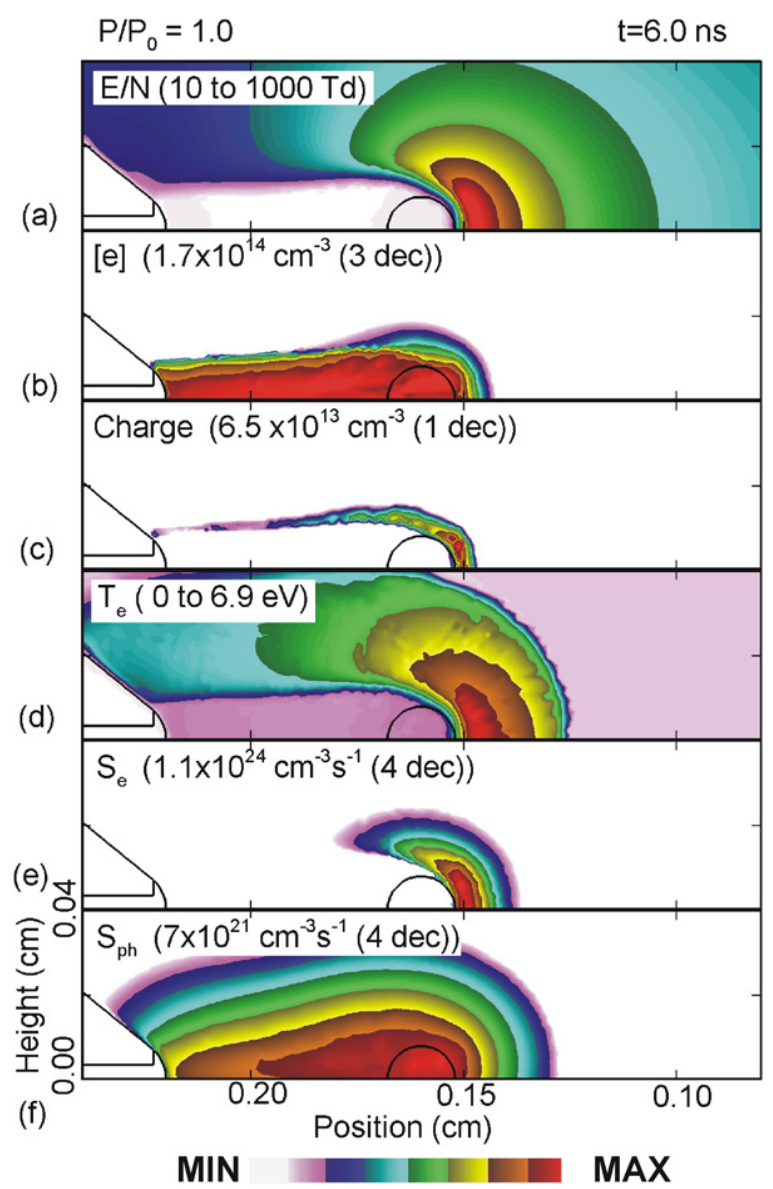

Figure 2. Plasma parameters for a streamer propagating in unperturbed atmospheric pressure air at $6.0 \mathrm{~ns}$ after the start of the $15 \mathrm{kV}$ pulse: $(a) E / N$, (b) $T_{\mathrm{e}}$, (c) electron density, $(d)$ positive space charge, $(e)$ electron impact ionization and $(f)$ photoionization source. The outline of the bubble is shown for reference to later results. The range of values or maximum value plotted are indicated in each frame. Log plots are indicated by the number of decades.

As such, the results discussed here and in Part II are best-case scenarios for the influence of bubbles on streamer propagation.

\section{Streamer characteristics in a homogeneous medium}

In order to provide a baseline, the properties of a positive corona streamer in humid atmospheric pressure air in the absence of inhomogeneities are briefly discussed. $E / N$, electron density, positive space charge (regions where the net charge is positive), $T_{\mathrm{e}}$, electron impact ionization source and photoionization source are shown in figure 2 during the propagation of the streamer. (The outline of a bubble to be placed below is shown for reference.) The streamer is initiated in the high electric field near the anode where the small radius of curvature produces geometric electric field enhancement. The streamer then moves toward the cathode, against the direction of electron drift, by seeding electrons ahead of the streamer by photoionization. The streamer can be divided into the head or avalanche front where $E / N$ is large due to charge separation and where ionization by electron impact occurs; and 
the high conductivity, low $E / N$ channel connecting the head with the anode. The plasma in the inner part of the channel is quasi-neutral with densities exceeding $10^{14} \mathrm{~cm}^{-3}$. Positive space charge is concentrated at the head of the streamer and at the boundaries of the streamer. The space charge at the head of the streamer produces an $E / N$ exceeding $1000 \mathrm{Td}$ $\left(1 \mathrm{Td}=10^{-17} \mathrm{~V} \mathrm{~cm}^{2}\right)$. Given the small radius of curvature of the streamer head, the electric field ahead of the streamer is geometrically enhanced, similar to that around the anode tip at the time of initiation. This electric field enhancement produces even larger electric fields, as the anode potential is compressed into the shorter, un-ionized gap ahead of the avalanche front. These large electric fields heat the electrons to temperatures as high as $7 \mathrm{eV}$.

Electron impact ionization occurs almost exclusively at the head of the streamer at rates exceeding $10^{24} \mathrm{~cm}^{-3} \mathrm{~s}^{-1}$ thereby propagating the streamer. Photon generation occurs both in the streamer head and in the conducting channel due to the finite lifetime of the excited states. It is the source of preionization ahead of the streamer. If photoionization is removed from the computation and there is no initial distribution of preionization, the streamer will stall and fail to propagate as it leaves the geometrically enhanced region of large $E / N$ near the electrode tip.

The diameter of the streamer is an important consideration in bubble-streamer interactions. Experimental observations and modeling have shown that the streamer radius is 100$200 \mu \mathrm{m}$, as we also obtain here [16]. The total propagation time of the streamer across the $2 \mathrm{~mm}$ gap is around $8 \mathrm{~ns}$, resulting in speeds of $2.5 \times 10^{7} \mathrm{~cm} \mathrm{~s}^{-1}$.

\section{Streamers intersecting with negative and positive bubbles}

Streamer properties when intersecting a negative bubble $80 \mu \mathrm{m}$ in radius on axis are shown in figures $3(E / N$, electron density and positive space charge) and 4 ( $T_{\mathrm{e}}$, electron impact ionization and photoionization sources). The pressure in the bubble is 700 Torr while the ambient $\left(P_{0}\right)$ pressure is atmospheric, giving a rarefaction of $P / P_{0}=0.92$. Due to its lower gas density, the $E / N$ inside the bubble is larger than the ambient gas. As the streamer approaches, there is a further increase in $E / N$ due to the geometric field enhancement ahead of the avalanche front. As photoionization seeds electrons in the bubble, the elevated $E / N$ produces a more rapid rate of ionization than in the ambient. This ionization produces a source of electrons that drift upstream into the gap between the bubble and the approaching avalanche front. Photoionization from the bubble also seeds electrons in the ambient gas near the bubble. As a result, the streamer accelerates into the bubble.

In this example, the streamer width is comparable to but larger than the size of the bubble. The streamer width decreases and the streamer converges into the bubble. After crossing through the negative bubble, the streamer regains the width it had before encountering the bubble and would have had in the absence of the bubble. As shown below, after traversing a bubble with a larger radius, the streamer may not regain its original properties. The electron density inside the bubble

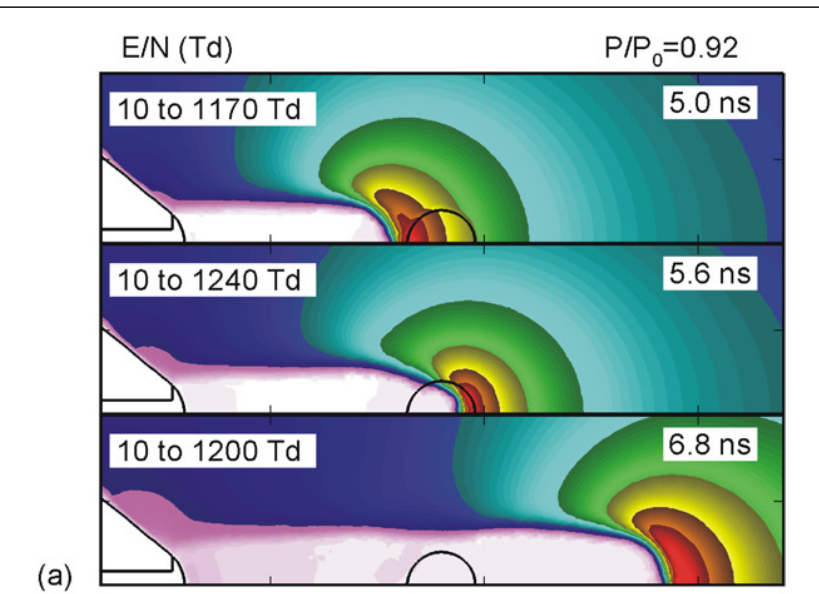

(a)
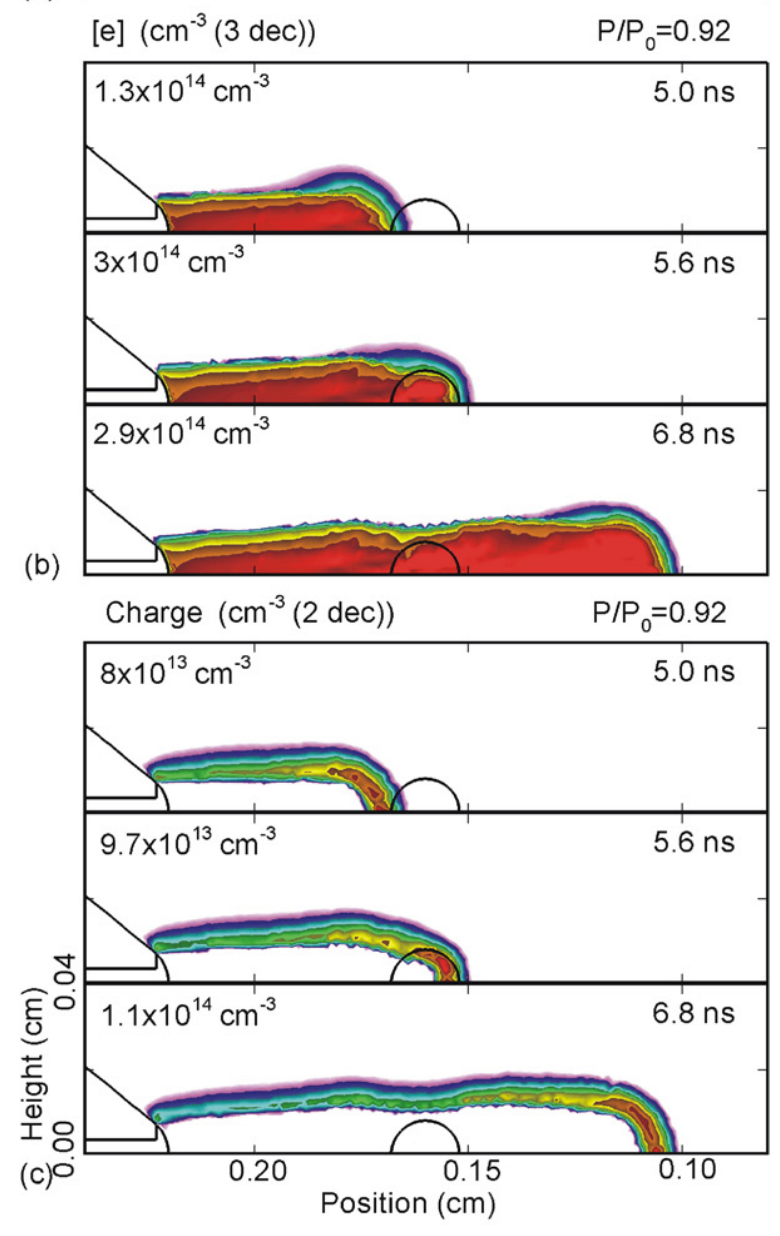

MIN

MAX

Figure 3. Plasma parameters for a streamer intersecting a $80 \mu \mathrm{m}$ negative bubble $\left(P / P_{0}=0.92\right)$ on the streamer axis at 5.0, 5.6 and $6.8 \mathrm{~ns}$ : (a) $E / N$, (b) electron density and (c) positive space charge. The streamer focuses into the bubble. No branching occurs. The range of values or maximum value plotted are indicated in each frame. Log plots are indicated by the number of decades.

increases up to $3 \times 10^{14} \mathrm{~cm}^{-3}$, a factor of two greater than in the absence of the bubble. After the streamer passes through the bubble, the electron density decreases to that of an unperturbed streamer. The more rapid production of electrons inside the bubble increases the propagation speed of the streamer on axis, giving the appearance of the streamer focusing into the bubble. The streamer tip has a convex shape. 
(a)

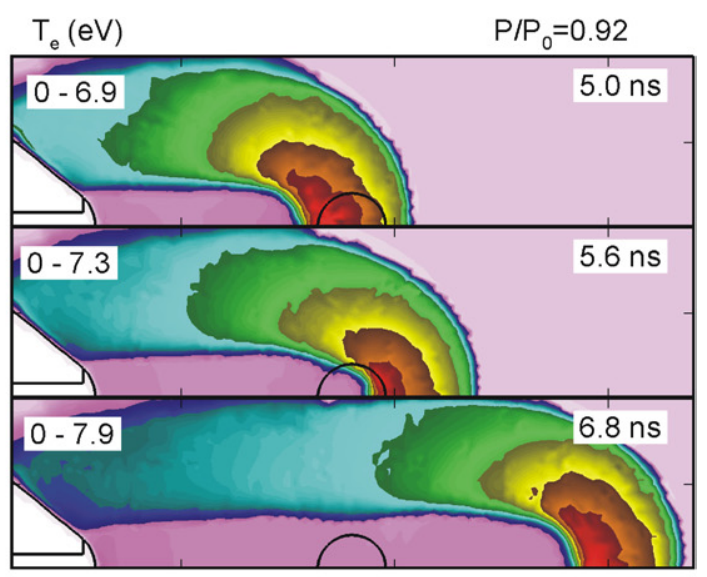

E-Impact $\left(\mathrm{cm}^{-3} \mathrm{~s}^{-1}(4 \mathrm{dec})\right)$

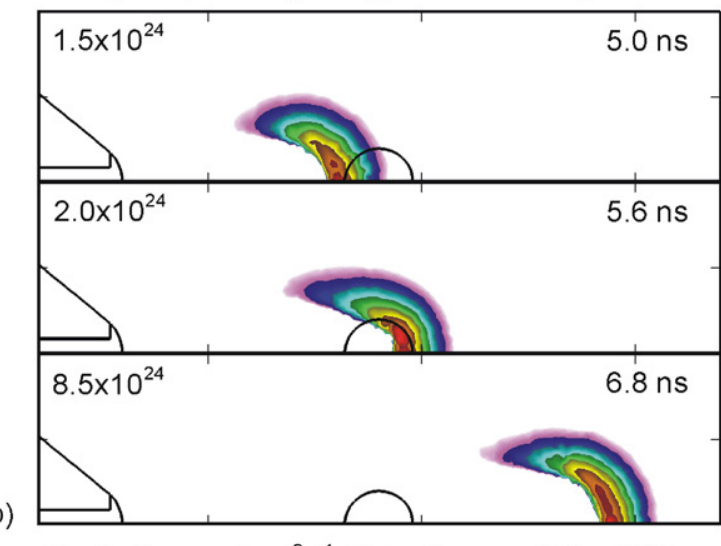

(b)
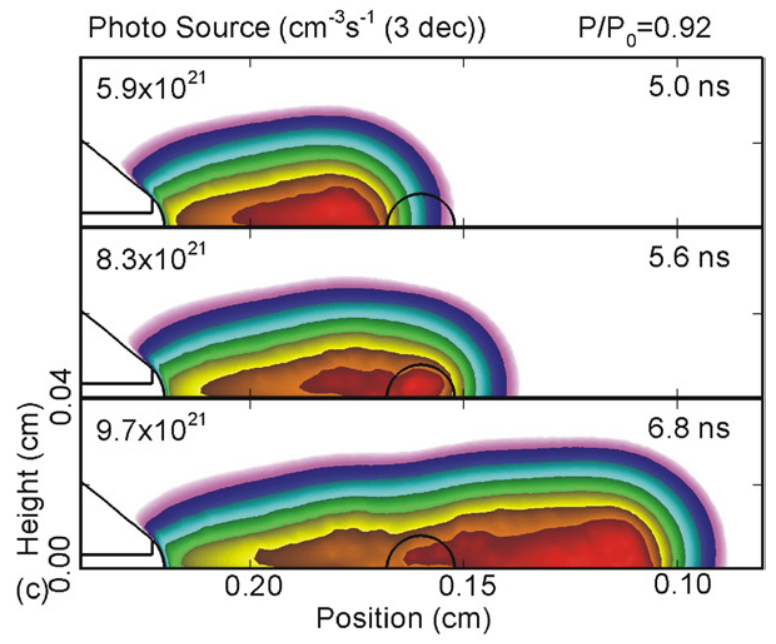

MIN

Figure 4. Plasma parameters for a streamer intersecting a $80 \mu \mathrm{m}$ negative bubble $\left(P / P_{0}=0.92\right)$ on the streamer axis at 5.0, 5.6 and $6.8 \mathrm{~ns}$ : (a) $T_{\mathrm{e}}$, (b) electron impact ionization and (c) photoionization. The range of values or maximum value plotted are indicated in each frame. Log plots are indicated by the number of decades.

The streamer behaves quite differently when approaching a positive bubble on the axis, as shown in figures $5(E / N$, electron density and positive space charge) and $6\left(T_{\mathrm{e}}\right.$, electron impact ionization and photoionization sources) for $P / P_{0}=$ 1.08. In this case $E / N$ is lower in the bubble than in the ambient gas prior to the streamer approaching the bubble. $E / N$ is now larger off axis outside the bubble. Provided the total absorption length is comparable to the bubble, photoionization

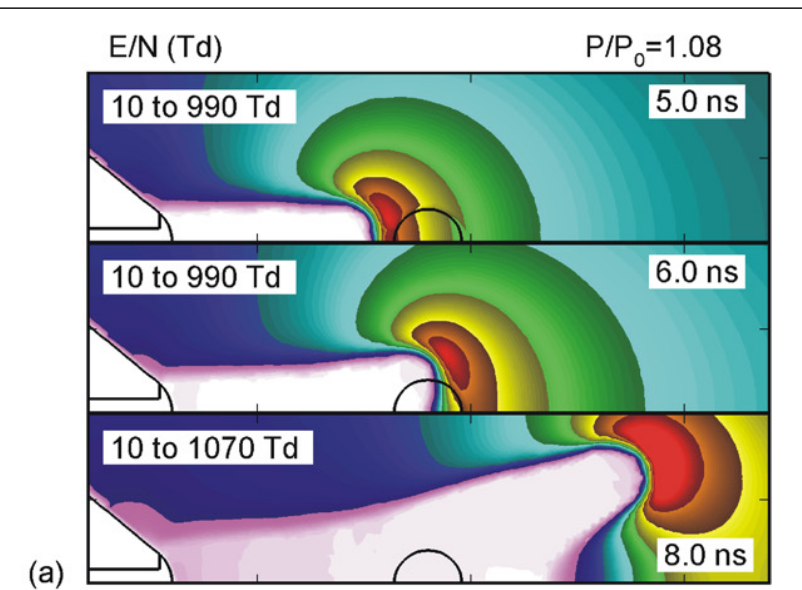

(a)

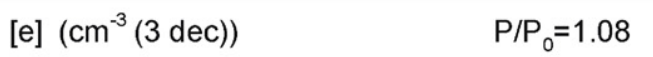

(b)
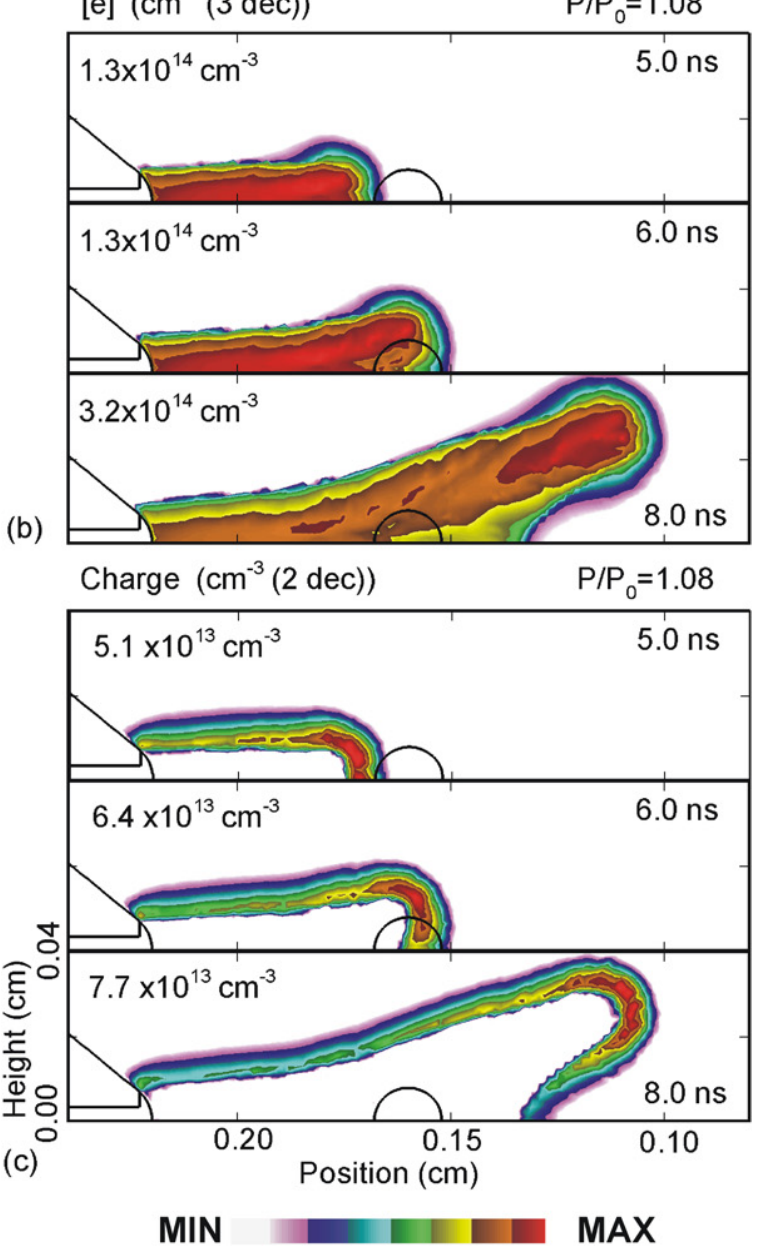

Figure 5. Plasma parameters for a streamer intersecting a $80 \mu \mathrm{m}$ positive bubble $\left(P / P_{0}=1.08\right)$ on the streamer axis at 5.0, 6.0 and $8.0 \mathrm{~ns}$ : $(a) E / N,(b)$ electron density and $(c)$ positive space charge. Streamer follows the path with larger rates of ionization, avoids the bubble rather than penetrating into it and streamer branching occurs. The range of values or maximum value plotted are indicated in each frame. Log plots are indicated by the number of decades.

seeds a larger density of electrons in the bubble than in the ambient because of its shorter absorption length in the higher density gas. However, the lower $E / N$ inside the bubble prevents their rapid ionization. Although there is plasma inside the bubble, the lower $E / N$ and lower ionization on axis causes the streamer to divert (around the bubble toward the region of 


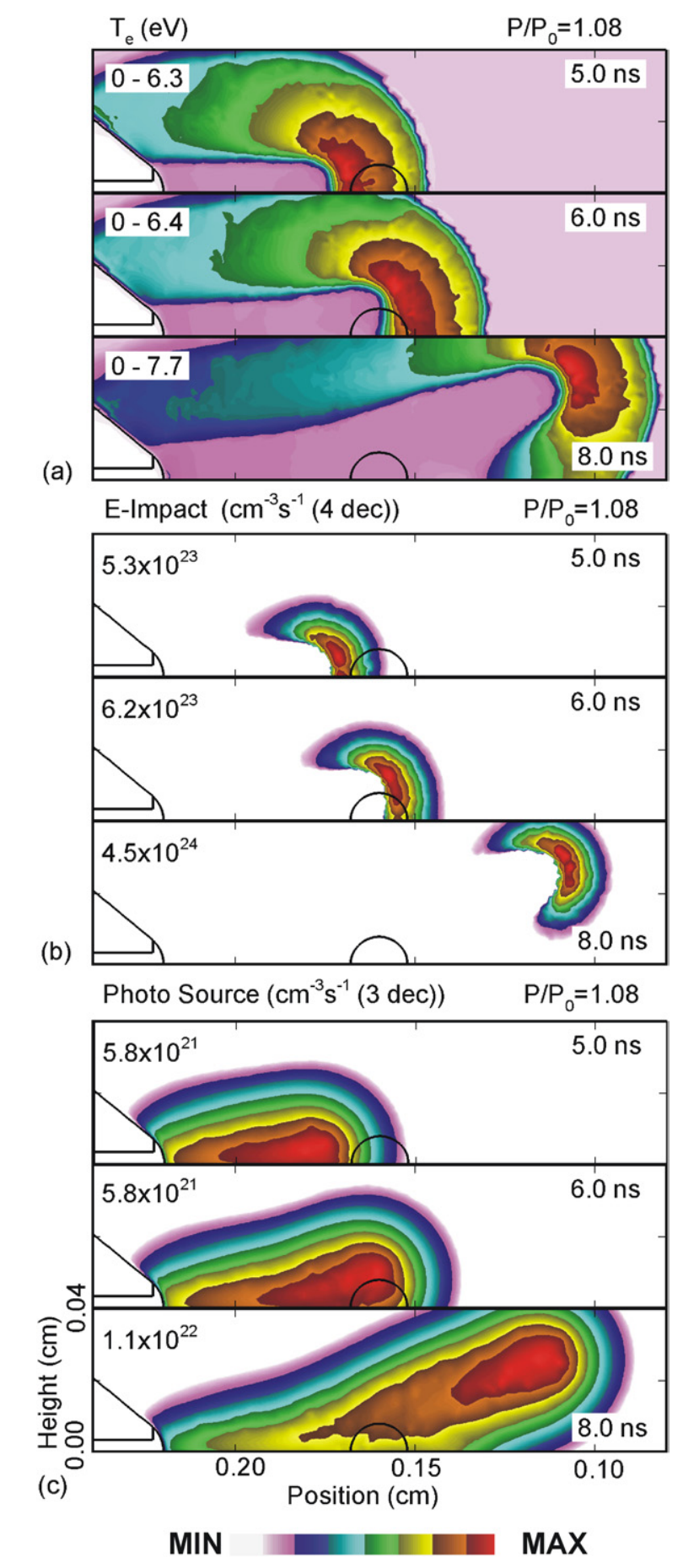

Figure 6. Plasma parameters for a streamer intersecting a $80 \mu \mathrm{m}$ positive bubble $\left(P / P_{0}=1.08\right)$ on the streamer axis at 5.0,6.0 and $8.0 \mathrm{~ns}$ : (a) $T_{\mathrm{e}},(b)$ electron impact ionization and (c) photoionization. The range of values or maximum value plotted are indicated in each frame. Log plots are indicated by the number of decades.

higher ionization on its periphery). For a bubble on the axis of symmetry, this diversion can also be described as branching since two streamers (one on either side of the axis of symmetry) are produced.

Once past the bubble, the streamer does not veer back to the axis where the $E / N$ would be largest in the absence of the bubble. The streamer continues on a branched trajectory driven by its own space charge enhanced electric field. In this case, the positive bubble acts as an obstacle in the streamer path. The lower $E / N$ inside the bubble compared with ambient results in a larger electron density on its periphery, which also increases the speed of the streamer in a path around the bubble. If the bubble is small enough, the avalanche head proceeds past the bubble prior to there being significant avalanche inside the bubble. This reduces the local voltage drop and so chokes off further ionization in the bubble. Streamer tip acquires a concave shape in passing the bubble. The end result is that it appears as though the streamer branches on the positive bubble.

Comparing the negative and positive bubbles, the larger $E / N$ in the negative bubble produces a $T_{\mathrm{e}}$ of $7.3 \mathrm{eV}$, whereas that in the positive bubble is $6.4 \mathrm{eV}$, with a larger peak ionization $\left(2.0 \times 10^{24}\right.$ compared with $\left.6.2 \times 10^{23} \mathrm{~cm}^{-3} \mathrm{~s}^{-1}\right)$. These differences and the different streamer behavior they instigate occur with relative changes of $E / N$ of only \pm 0.08 . The degree of focusing (negative bubble) or deflection (positive bubble) depends on the magnitude of these deviations in $E / N$ and the mean free path of photoionizing radiation $\lambda_{\mathrm{P}}$. For $\lambda_{\mathrm{P}}=220 \mu \mathrm{m}$, as used here, we found that $P / P_{0}<0.94$ will produce focusing and $P / P_{0} \geqslant 1.065$ will produce branching. As discussed below, other conditions being equal, a larger $\lambda_{P}$ will produce a larger streamer width. If the width is larger than the size of the inhomogeneity branching will not occur.

\section{Streamer characteristics with solid particles}

We found for the conditions considered here, a streamer may appear to branch when encountering a positive bubble with only a small pressure increment. As a limiting case of a very dense bubble, one might expect that a solid bubble (i.e. a particle) would act as an efficient branching agent. That said, the interaction of a streamer tip with its enhanced electric field with a solid particle is more complicated due to the resulting polarization. For example, the polarization electric field for a dielectric sphere of constant permittivity $\varepsilon$ with radius $r_{0}$ in a uniform external electric field $\vec{E}_{0}$ aligned with the polar axis is [35]

$$
\begin{aligned}
\vec{E}= & E_{0} \cos \theta\left[1+2\left(\frac{r_{0}}{r}\right)^{3} \frac{\varepsilon-1}{\varepsilon+2}\right] \hat{a}_{r} \\
& -E_{0} \sin \theta\left[1-\left(\frac{r_{0}}{r}\right)^{3} \frac{\varepsilon-1}{\varepsilon+2}\right] \hat{a}_{\theta} .
\end{aligned}
$$

The electric field at the poles $(\theta=0, \pi)$ of the particle is enhanced by a factor of $1+2(\varepsilon-1) /(\varepsilon+2)$ compared with the unperturbed applied electric field. At the equator $(\theta=\pi / 2)$ the electric field at the surface of the particle is decreased by a factor of $1-(\varepsilon-1) /(\varepsilon+2)$. Even in a uniform external electric field, the polarization of the particle may produce non-uniform rates of ionization as a function of azimuth. Expressions analogous to equation (6) cannot, in general, be obtained analytically if the electric field is non-uniform and transient. In the model, the polarization electric fields are a natural outcome of the solution of Poisson's equation in both the plasma and the particles while including all of the appropriate material properties and charges [28].

Streamer dynamics with a solid particle $80 \mu \mathrm{m}$ in radius on axis with $\varepsilon / \varepsilon_{0}=2.5$ are shown in figures $7(E / N$, electron 


\section{Solid particle $\varepsilon=2.5$}

$\mathrm{E} / \mathrm{N}(\mathrm{Td})$

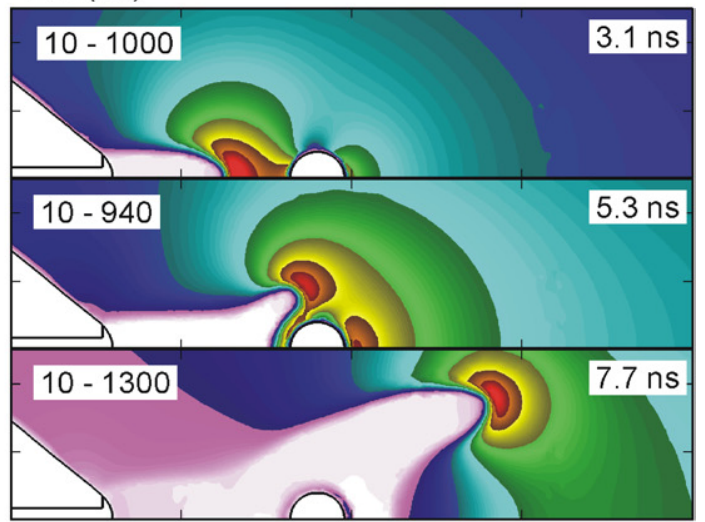

(a)

[e] $\left(\mathrm{cm}^{-3}(3 \mathrm{dec})\right)$

(b)

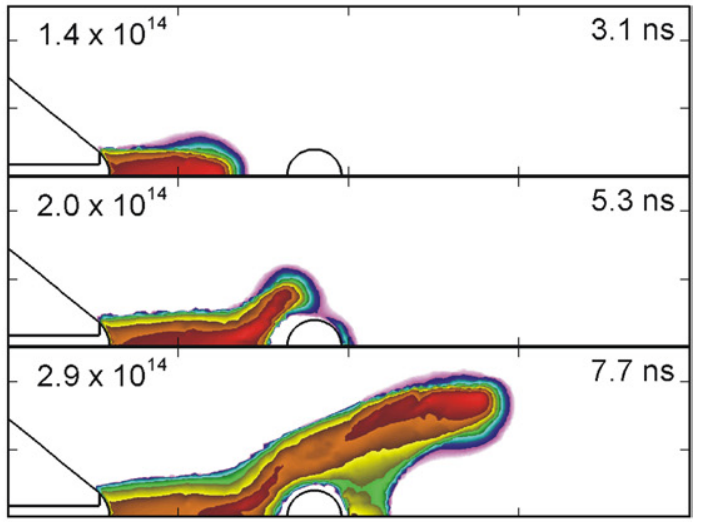

Charge $\left(\mathrm{cm}^{-3}(2 \mathrm{dec})\right)$

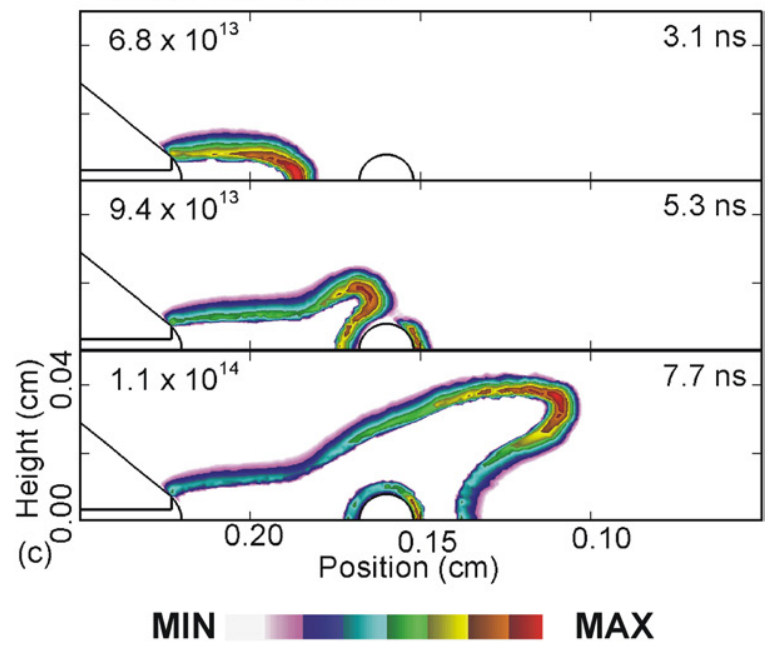

Figure 7. Plasma parameters for a streamer intersecting a low permittivity $80 \mu \mathrm{m}$ solid particle $\left(\varepsilon / \varepsilon_{0}=2.5\right)$ on the streamer axis at 3.1, 5.3 and $7.7 \mathrm{~ns}:(a) E / N,(b)$ electron density and (c) positive space charge. The streamer charges the low capacitance particle and branches around it. The range of values or maximum value plotted are indicated in each frame. Log plots are indicated by the number of decades.

density $n_{\mathrm{e}}$ and positive space charge $)$ and $8\left(T_{\mathrm{e}}\right.$, electron impact ionization and photoionization sources). As the streamer approaches the particle and its plasma begins to envelope the particle, ionizing photons reach the right side of the particle that previously was in the shadow. For this low permittivity (a)

$$
\mathrm{T}_{\mathrm{e}}(\mathrm{eV})
$$

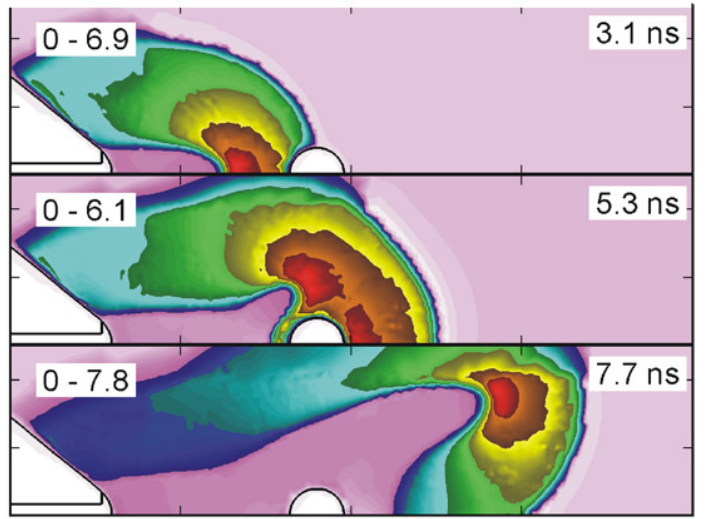

E-Impact $\left(\mathrm{cm}^{-3} \mathrm{~s}^{-1}(4 \mathrm{dec})\right)$
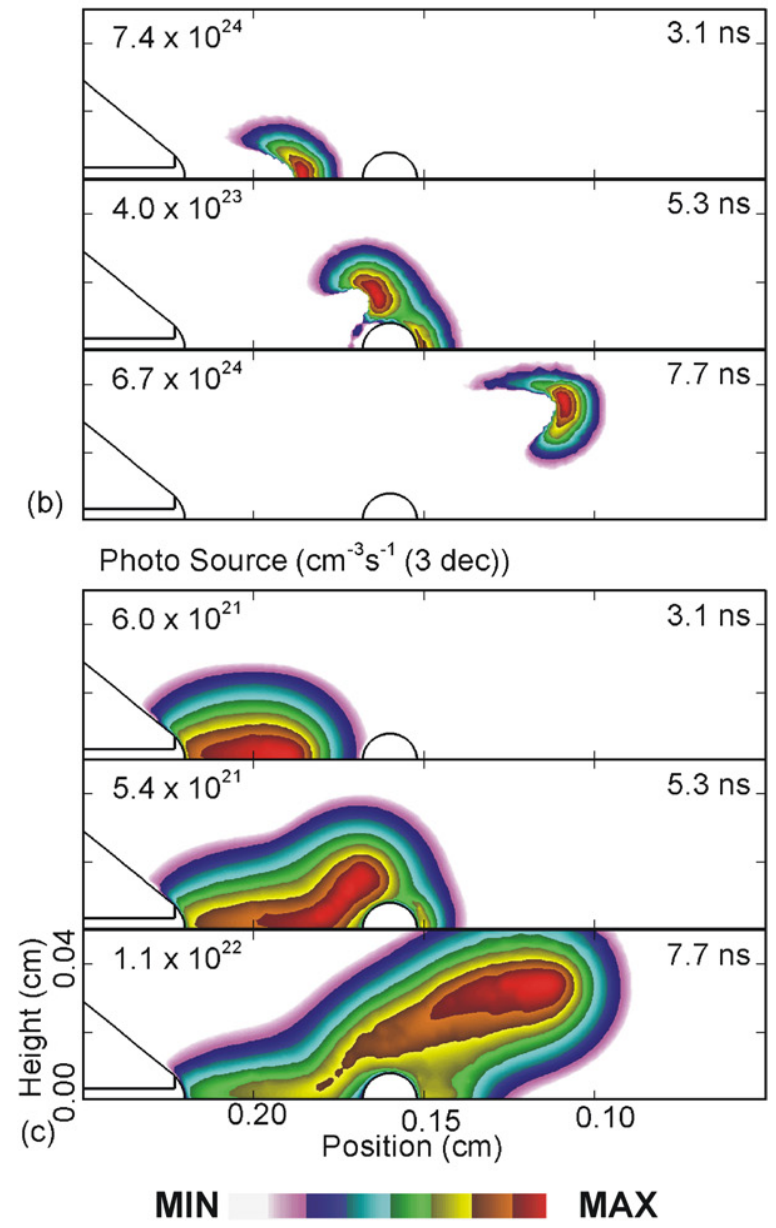

Figure 8. Plasma parameters for a streamer intersecting a low permittivity $80 \mu \mathrm{m}$ solid particle $\left(\varepsilon / \varepsilon_{0}=2.5\right)$ on the streamer axis at 3.1, 5.3 and $7.7 \mathrm{~ns}:(a) T_{\mathrm{e}}$, (b) electron impact ionization and $(c)$ photoionization. Log plots are indicated by the number of decades.

particle, there is a factor of 1.7 enhancement in the electric field at the right pole. The electrons produced by photoionization in this region of larger $E / N$ avalanche, producing the initial stage of launching a streamer from the right side of the particle. Simultaneously, the incoming streamer charges the particle which produces a deflecting electric field that guides the streamer around the particle. Further streamer 
dynamics depend on the competition between the primary streamer enveloping and deflecting around the particle, and the development of a daughter streamer from the right side of the particle.

For small permittivities $\left(\varepsilon / \varepsilon_{0}<2.5\right)$ there is a small enhancement in $E / N$ by the polarization fields. As a result, the avalanche at the right side of the particle launching the daughter streamer is slow. The primary streamer charges the particle and is deflected around the particle before the daughter streamer can be fully launched. The deflected streamer does not move back toward the axis but propagates in its own space charge field enhanced $E / N$. This gives the appearance of a branching event. Note that the streamer deposits positive charge on the particle with the exception of the right pole which acquires a negative charge from electrons drifting back toward the anode. This is the result of the transient nature of the interaction. Over a longer time scale (up to $1 \mathrm{~ms}$ ) the particle will acquire a large negative charge in the decaying plasma of the streamer channel [36].

The scenario is different when the streamer intersects high permittivity particle, $\varepsilon / \varepsilon_{0}=25$, as shown in figures $9(E / N$, electron density and positive space charge $)$ and $10\left(T_{\mathrm{e}}\right.$, electron impact ionization and photoionization sources). When the streamer intersects the high permittivity particle, there is proportionately more polarization at its poles producing larger $E / N$. The capacitance of the particle is also larger. As a result, the streamer stalls upon intersecting the particle as opposed to immediately diverging around the particle. The stalling is a result of charge being pulled out of the streamer to charge the particle and the larger $E / N$ at the pole that provides local ionization. As the plasma begins to spread around and envelope the particle (perhaps a precursor to branching), photoionization seeds electrons in the high electric field at the right pole of the particle. This pre-ionization launches a new streamer in the large $E / N$ at the pole. This daughter streamer is not initially connected by plasma to the primary streamer intersecting at the left pole of the particle. As this new streamer begins to propagate and carry with it a large potential drop across its head, the particle and primary streamer are left in low electric field regions. The primary streamer finally stalls out in its early stage of branching.

The intersection of a streamer with a pure, de-ionized (that is, low conductivity) water droplet, having $\varepsilon / \varepsilon_{0}=80$, should follow the same trends as that for the $\varepsilon / \varepsilon_{0}=25$ case. However the correspondence will not be 1-to-1 for a water droplet having finite conductivity due to the possible dissipation of charge and the charging of the interface from the interior of the droplet. These issues will be discussed in a forthcoming publication.

\section{Bubble size and location}

In general, streamer-bubble dynamics are functions of bubble size compared with the streamer width. The streamer can envelope a bubble smaller than its width without there being a significant change in its structure provided $P / P_{0}$ is sufficiently close to unity. The larger the deviation of $P / P_{0}$ from unity, the more influential a bubble of a given radius is on the streamer

\section{Solid particle $\varepsilon=25$}

$\mathrm{E} / \mathrm{N}(\mathrm{Td})$

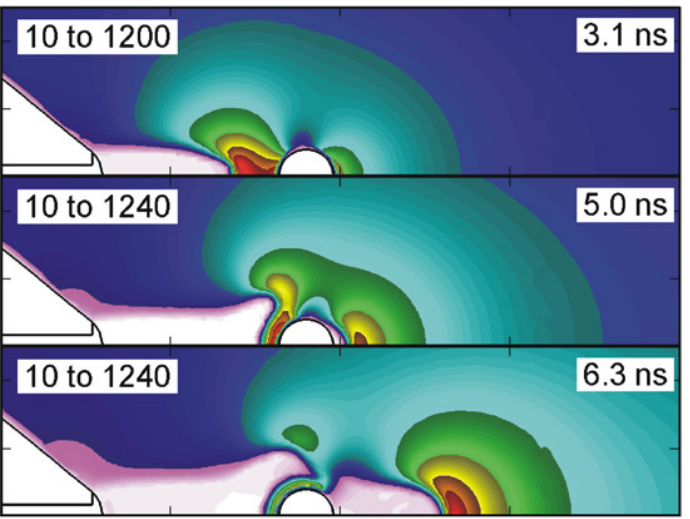

(a)

[e] $\left(\mathrm{cm}^{-3}(3 \mathrm{dec})\right)$
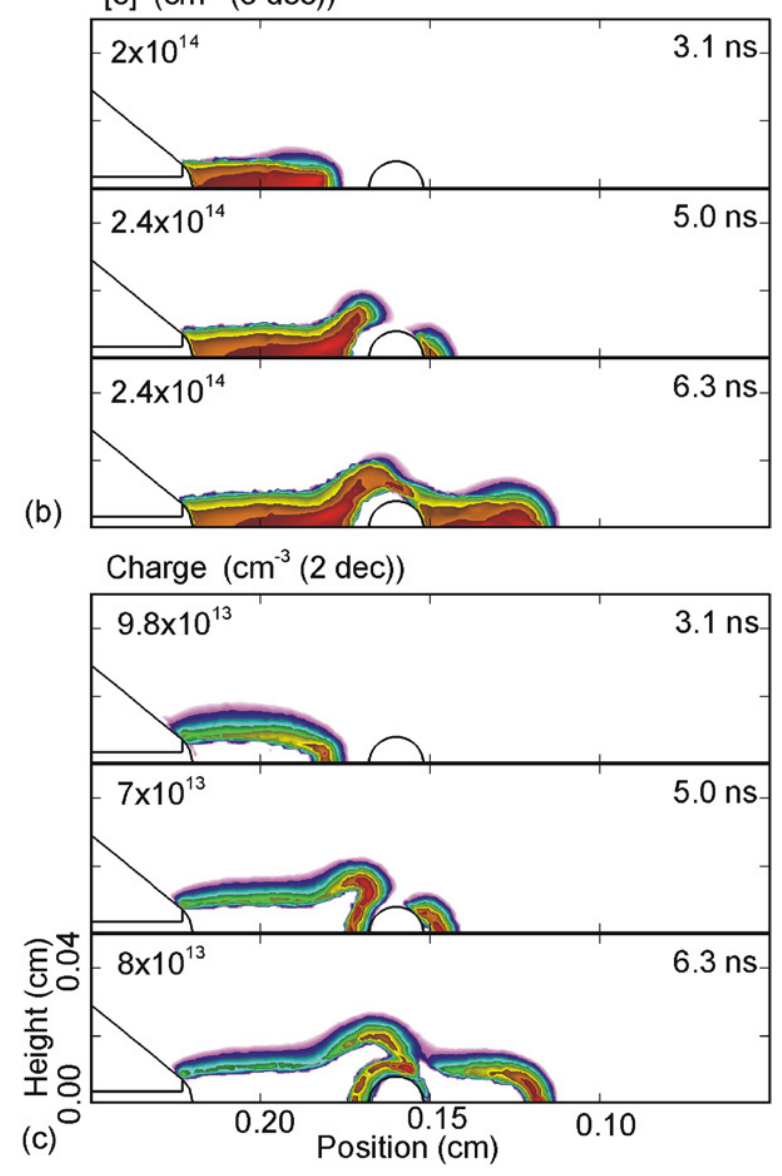

MIN

MAX

Figure 9. Plasma parameters for a streamer intersecting a high permittivity $80 \mu \mathrm{m}$ solid particle $\left(\varepsilon / \varepsilon_{0}=25\right)$ on the streamer axis at 3.1, 5.0 and $6.3 \mathrm{ns:}(a) E / N$, (b) electron density and (c) positive space charge. The range of values or maximum value plotted are indicated in each frame. Log plots are indicated by the number of decades.

dynamics. For example, as discussed in Part II [29], negative bubbles smaller than the streamer can perturb the streamer provided $P / P_{0}$ is small enough.

Streamer properties intersecting negative bubbles $\left(P / P_{0}=0.92\right)$ having radii of 40,80 and $320 \mu \mathrm{m}$ are shown 


\section{Solid particle $\varepsilon=25$}

$\mathrm{T}_{\mathrm{e}}(\mathrm{eV})$

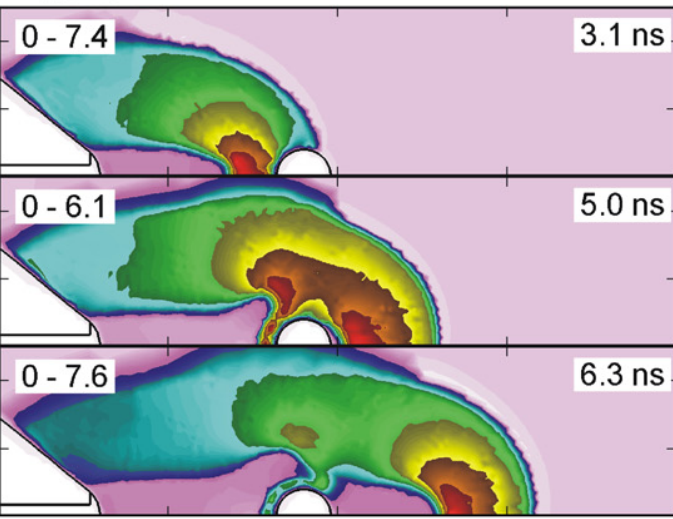

(a)

E-Impact $\left(\mathrm{cm}^{-3} \mathrm{~s}^{-1}(4 \mathrm{dec})\right)$

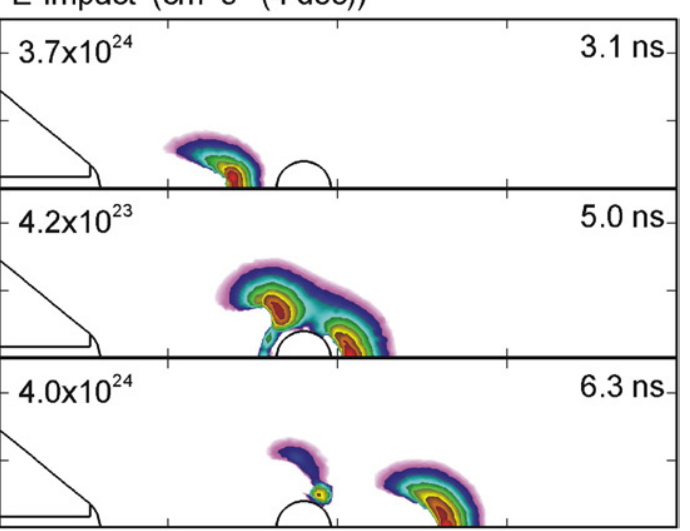

(b)

Photo Source $\left(\mathrm{cm}^{-3} \mathrm{~s}^{-1}(3 \mathrm{dec})\right)$

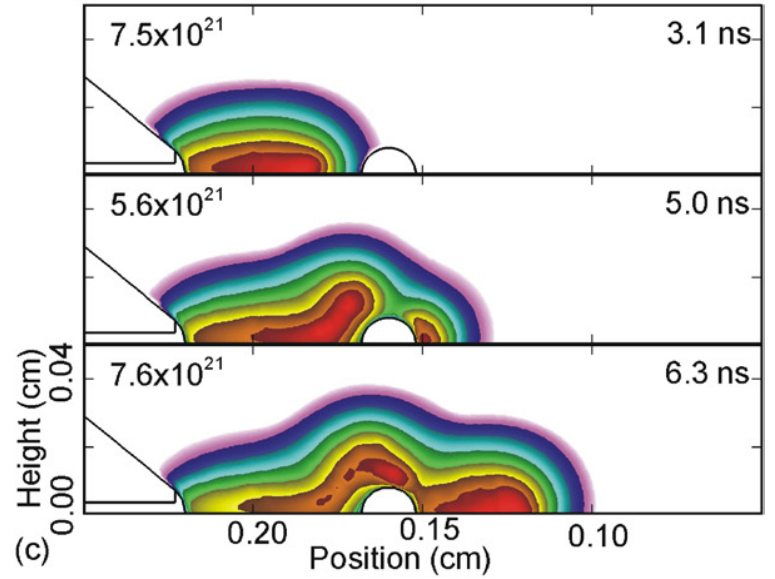

MIN

Figure 10. Plasma parameters for a streamer intersecting a high permittivity $80 \mu \mathrm{m}$ solid particle $\left(\varepsilon / \varepsilon_{0}=25\right)$ on the streamer axis at 3.1, 5.0 and $6.3 \mathrm{ns:}(a) T_{\mathrm{e}},(b)$ electron impact ionization and $(c)$ photoionization. The streamer stalls as it intersects the high capacitance particle. Photoelectrons produced in the large $E / N$ at the right pole initiates a daughter streamer. The range of values or maximum value plotted are indicated in each frame. Log plots are indicated by the number of decades.

in figures 11 (positive space charge) and 12 (electron density). Bubbles with radii much less than streamer width do not significantly perturb the streamer. Negative bubbles comparable in size to the streamer tend to focus and narrow the streamer by

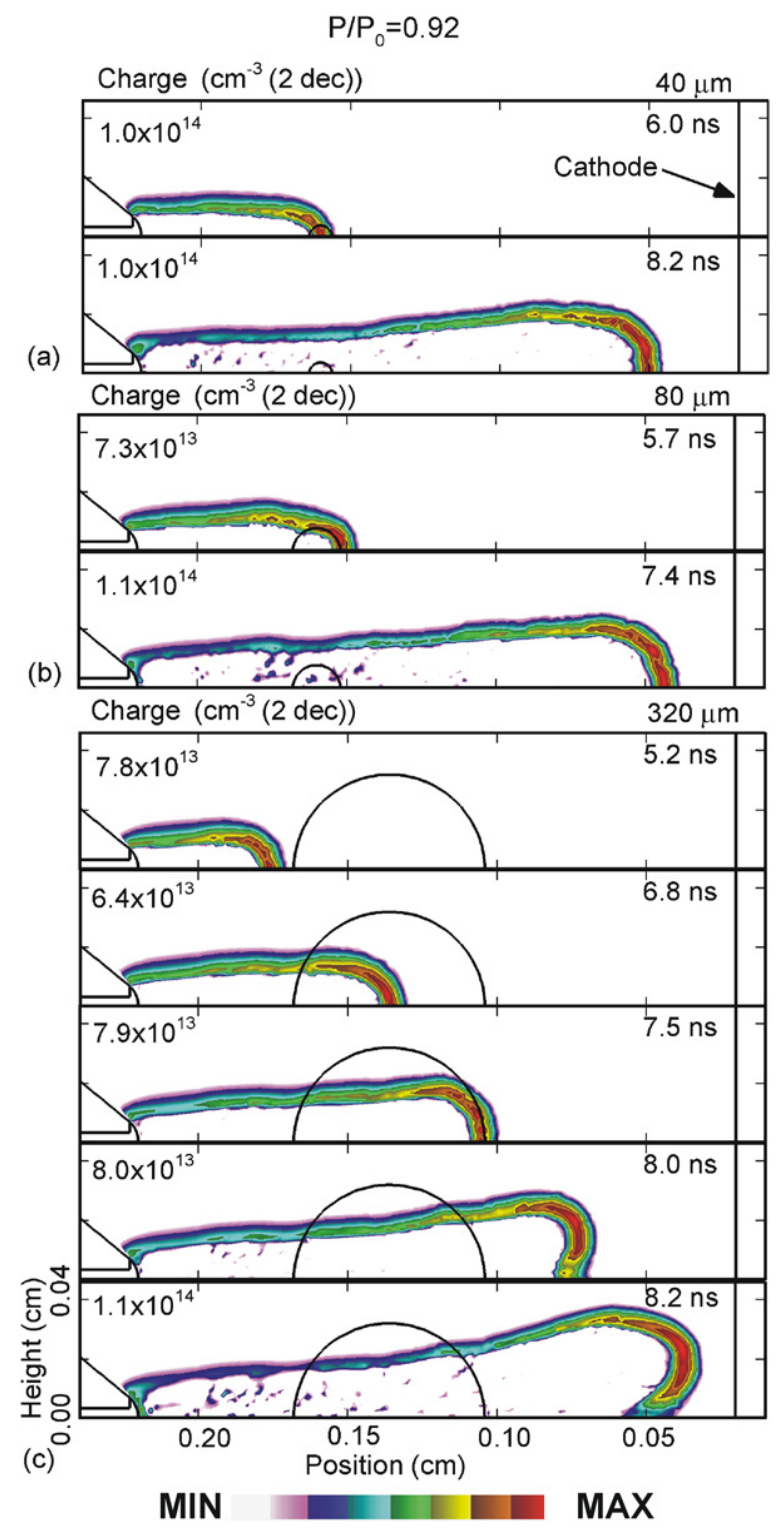

Figure 11. Positive space charge for a streamer intersecting a negative bubble $\left(P / P_{0}=0.92\right)$ for $(a) 40,(b) 80$ and $(c) 320 \mu \mathrm{m}$ in radius. Streamer dynamics are not significantly influenced by small bubbles. Branching on the right side of large bubbles occurs due to curvature distortion of the streamer front. The maximum value plotted is indicated in each frame for log plots over 2 decades.

virtue of the increased ionization and plasma density inside the bubble, which fills the entire volume of the bubble. After propagating through and around the bubble, the streamer continues as if unperturbed.

If the negative bubble is larger than the width of the streamer, the incident streamer penetrates into the bubble. Once inside the bubble, the streamer radius increases in the lower pressure (streamer radii scale approximately inversely with pressure) $[9,10]$. However, the streamer plasma does not necessarily occupy the entire volume of the bubble. In this case the left boundary of the bubble does not appreciably affect streamer propagation other than increasing its speed. Upon exiting the bubble at the right boundary, the curvature of the bubble enables the broader streamer to first cross into 
(a)

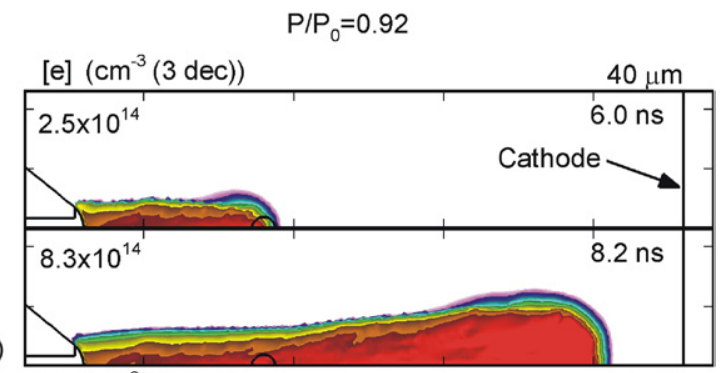

(b)
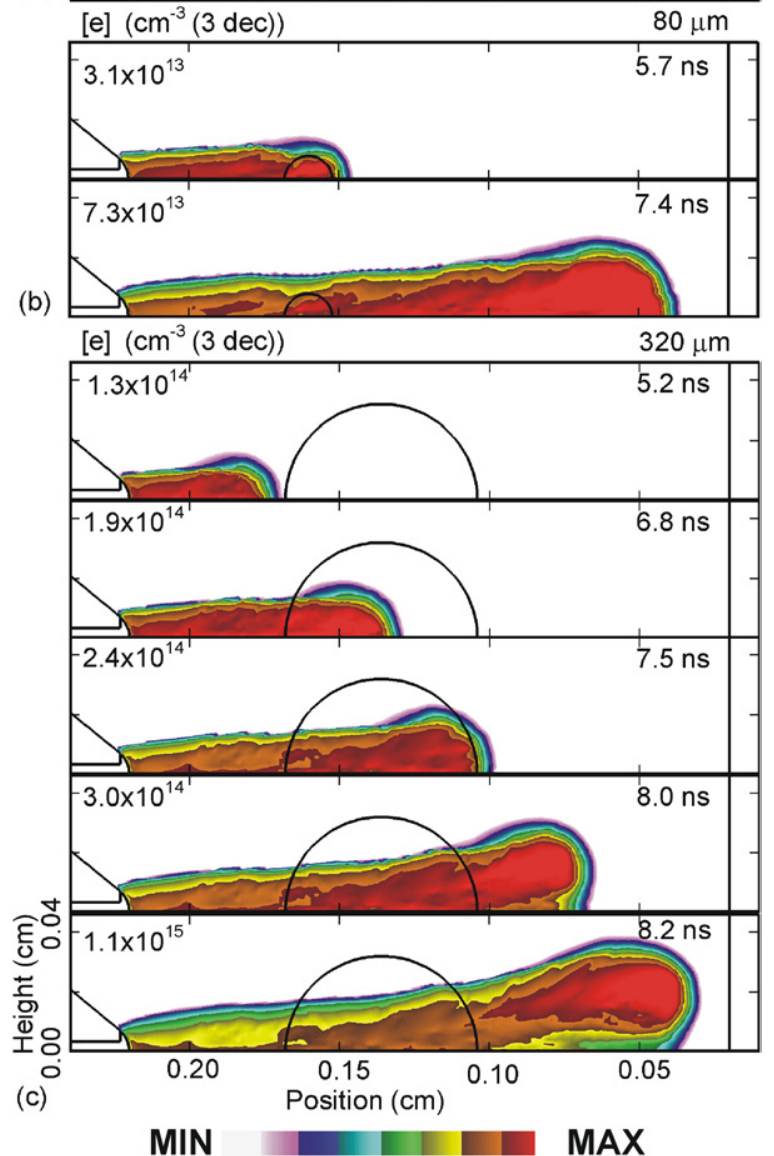

Figure 12. Electron density for a streamer intersecting a negative bubble $\left(P / P_{0}=0.92\right)$ for $(a) 40,(b) 80$ and (c) $320 \mu \mathrm{m}$ in radius. The maximum value plotted is indicated in each frame for log plots over 2 decades.

the ambient gas off axis. Some charge accumulation on axis at the bubble boundary in transitioning into the lower mobility ambient gas retards the on axis portion of the streamer. As a result of this distortion, the streamer branches at the right boundary of the negative bubble.

Streamer properties intersecting positive bubbles $\left(P / P_{0}=\right.$ 1.08 ) of 40,80 and $320 \mu \mathrm{m}$ are shown in figures 13 (positive space charge) and 14 (electron density). Streamers intersecting with positive bubbles of small radii are not significantly perturbed whereas intersecting with positive bubbles of comparable radii produce branching. The bubble, with its lower $E / N$ and lower rate of ionization, acts like an obstacle to the streamer. The streamer tip intersecting the bubble first experiences this smaller $E / N$ while the outer portion of the streamer is still in a region of larger $E / N$. An off axis streamer is launched and is sustained by its own space charge.

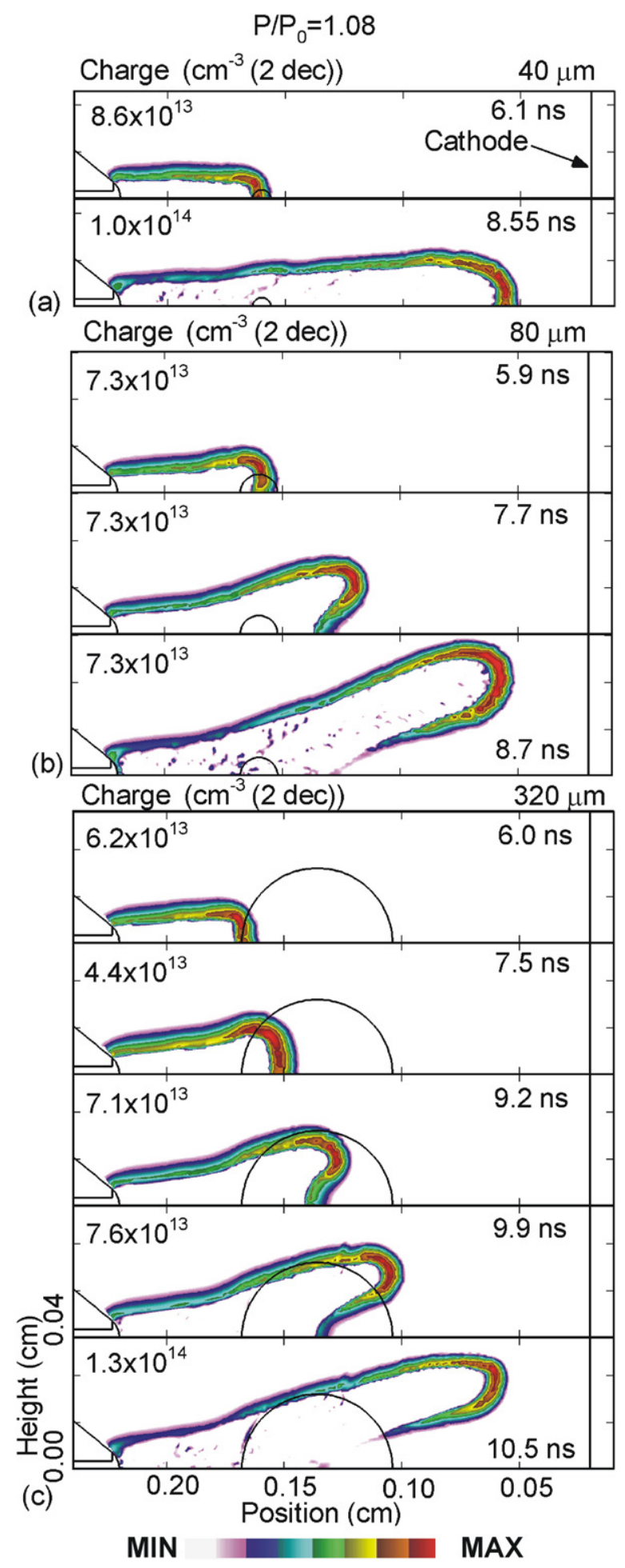

Figure 13. Positive space charge for a streamer intersecting a positive bubble $\left(P / P_{0}=1.08\right)$ for $(a) 40,(b) 80$ and (c) $320 \mu \mathrm{m}$ in radius. Streamer dynamics are not significantly influenced by small bubbles. However with larger bubbles, the streamer slides along the bubble boundary, moderately penetrates the bubble, and finally branches on the left boundary. The maximum value plotted is indicated in each frame for log plots over 2 decades.

If the positive bubble is large enough, the streamer can penetrate into and through the bubble. In doing so the speed of the streamer decreases commensurate with the lower $E / N$ in the bubble. For example, the streamer requires $2.3 \mathrm{~ns}$ to 


$$
P / P_{0}=1.08
$$

[e] $\left(\mathrm{cm}^{-3}(2 \mathrm{dec})\right)$

\begin{tabular}{|c|c|}
\hline $1.9 \times 10^{14}$ & $\begin{array}{c}6.1 \mathrm{~ns} \\
\end{array}$ \\
\hline $7.0 \times 10^{14}$ & Cathode \\
\hline & $8.55 \mathrm{~ns}$ \\
\hline
\end{tabular}

(a)

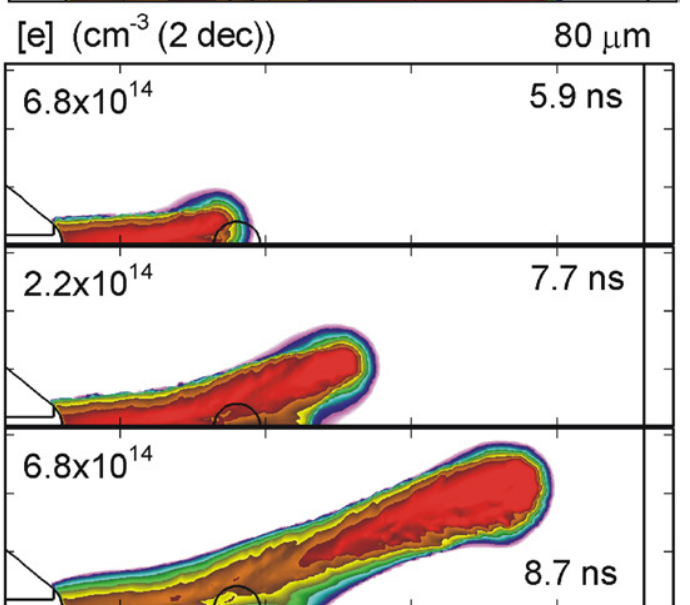

(b)

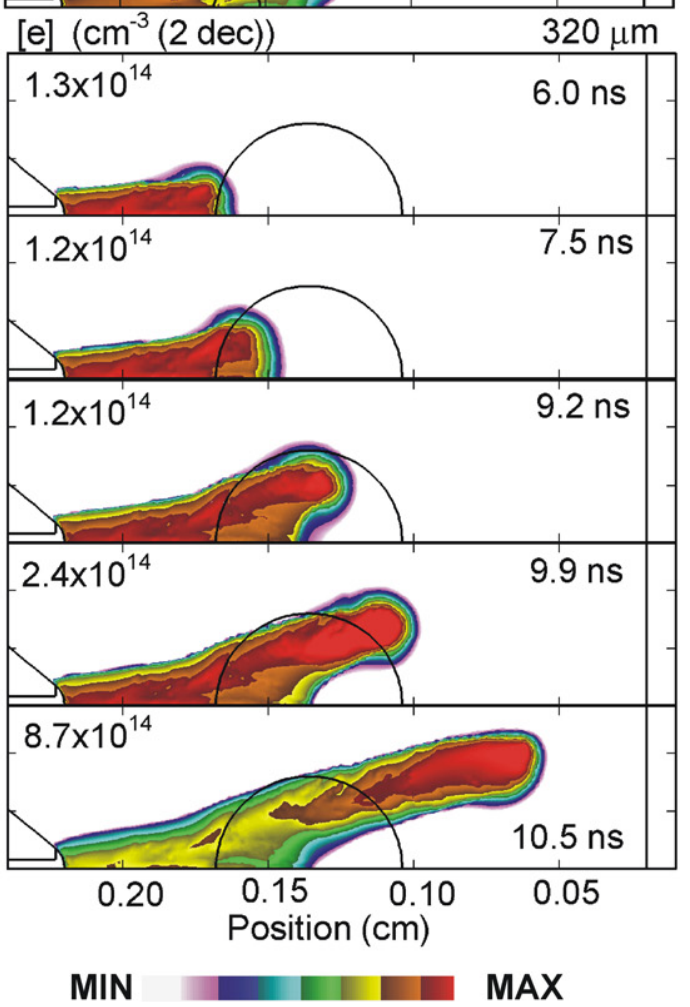

Figure 14. Electron density for a streamer intersecting a positive bubble $\left(P / P_{0}=1.08\right)$ for $(a) 40,(b) 80$ and $(c) 320 \mu \mathrm{m}$ in radius. The maximum value plotted is indicated in each frame for log plots over 2 decades.

traverse a negative bubble $\left(P / P_{0}=0.92\right) 320 \mu \mathrm{m}$ in radius $\left(1.4 \times 10^{7} \mathrm{~cm} \mathrm{~s}^{-1}\right)$ while requiring $3.9 \mathrm{~ns}$ to traverse the positive bubble $\left(P / P_{0}=1.08\right)$ of the same size $\left(8.2 \times 10^{6} \mathrm{~cm} \mathrm{~s}^{-1}\right)$. In this case, the streamer branches at the left boundary of the bubble, where the streamer front is first retarded on axis. In an effort to avoid the dense bubble, the streamer slides along

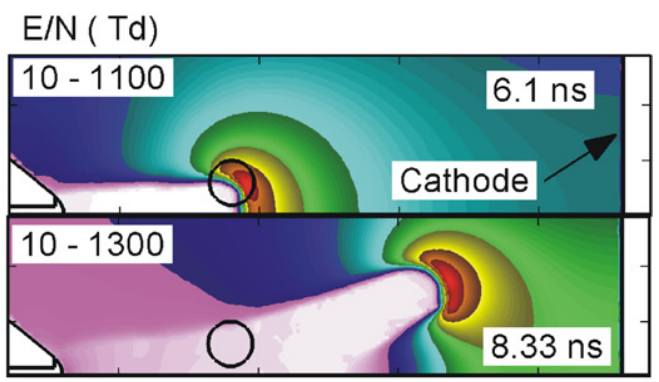

(a)

$$
\text { [e] }\left(\mathrm{cm}^{-3}(3 \mathrm{dec})\right)
$$

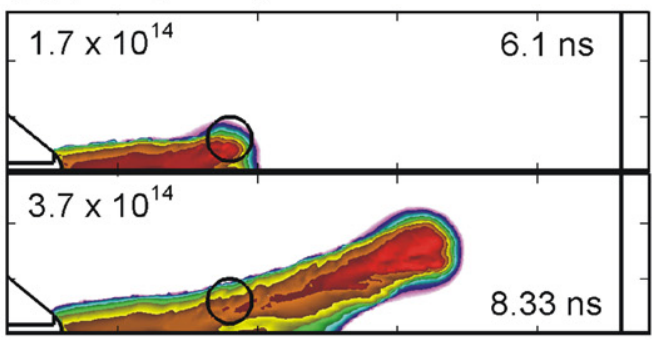

(b)

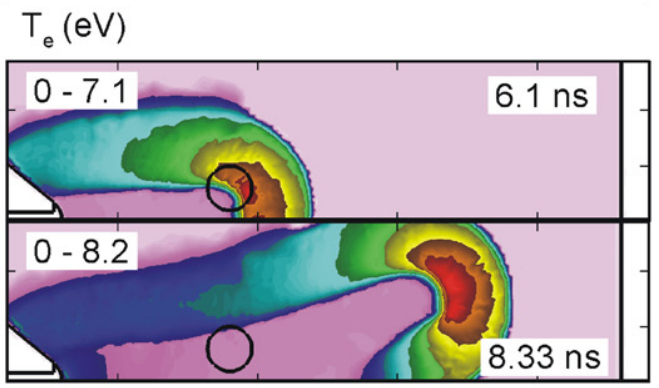

(c)

E-Impact $\left(\mathrm{cm}^{-3} \mathrm{~s}^{-1}(4 \mathrm{dec})\right)$

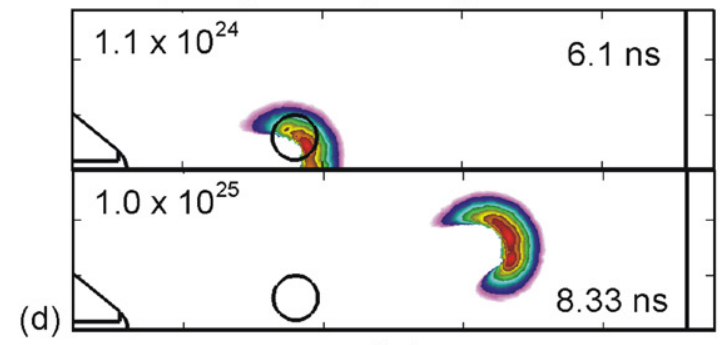

Photo Source $\left(\mathrm{cm}^{-3} \mathrm{~s}^{-1}(3 \mathrm{dec})\right)$

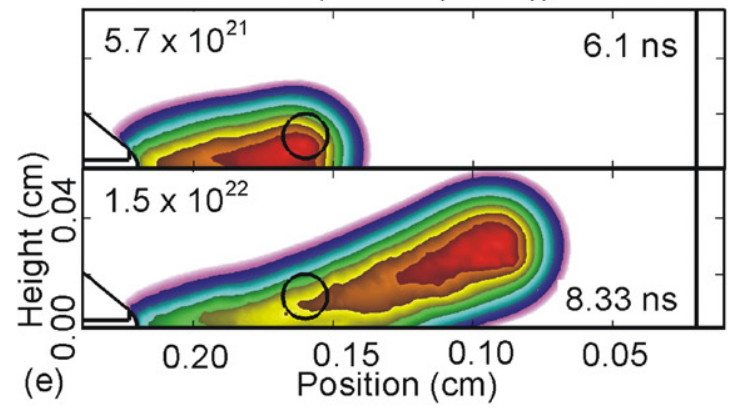

MIN

\section{MAX}

Figure 15. Plasma properties for a streamer approaching a negative bubble $\left(P / P_{0}=0.92\right)$ of $80 \mu \mathrm{m}$ radius centered $120 \mu \mathrm{m}$ off axis at 6.1 and 8.3 ns: (a) $E / N$, (b) electron density, $(c) T_{\mathrm{e}},(d)$ electron impact ionization and $(e)$ photoionization sources. Precursor electrons produced by photoionization are avalanched more rapidly in the larger $E / N$ inside the bubble resulting in the streamer branching. The range of values or maximum value plotted are indicated in each frame. Log plots are indicated by the number of decades. 
the bubble boundary where $E / N$ is larger and finally branches while moderately penetrating the bubble.

In naturally occurring conditions, it is not likely that a bubble occurs exactly on the streamer axis, and so we investigated the consequences of bubbles located off axis. Streamer properties in the vicinity of an $80 \mu \mathrm{m}$ negative bubble $\left(P / P_{0}=0.92\right)$ centered $120 \mu \mathrm{m}$ off axis are shown in figure $15,\left(E / N\right.$, electron density, $T_{\mathrm{e}}$, electron impact ionization sources and photoionization). Properties for a positive bubble $\left(P / P_{0}=1.078\right)$ are shown in figure 16 . With the off axis negative bubble, precursor electrons produced by photoionization from the primary streamer are avalanched in the larger $E / N$ inside the bubble. $T_{\mathrm{e}}$ inside the bubble when the streamer tip approaches is $7.1 \mathrm{eV}$ compared with $6.0 \mathrm{eV}$ in front of the streamer on axis. These hotter electrons produce photoionizing radiation in the gap between the bubble and main streamer as well as providing a source of electrons which drift back toward the streamer. The main streamer tends to follow this path of seed electrons and, as a result, branching occurs. The divergence of the streamer is indicated by the wholly offaxis peaks in $T_{\mathrm{e}}$ and ionization source in the bubble as the streamer propagates through it.

A positive bubble located off axis, as shown in figure 16, presents a region of reduced $E / N$ compared to that on axis. This produces a lower $T_{\mathrm{e}}(5.0 \mathrm{eV})$ and smaller ionization source. In spite of there being locally larger photoionization sources in the bubble due to its shorter absorption length, the lower $E / N$ prevents significant avalanche. The streamer is unable to penetrate into the positive bubble and so is squeezed into the axis. There is a decrease in width and in speed of the streamer. The decrease in width produces a local increase of the electric field. Conservation of current requires that there be an increase in conductivity or electric field (or both) to push the current through the narrower gap. For example, $E / N$ at the streamer tip is $1100 \mathrm{Td}$ approaching the negative bubble and $1300 \mathrm{Td}$ for the positive bubble. The corresponding values of electron density and $T_{\mathrm{e}}$ are $1.7 \times 10^{14} \mathrm{~cm}^{-3}$ and $7.1 \mathrm{eV}$ for the negative bubble and $3.7 \times 10^{14} \mathrm{~cm}^{-3}$ and $7.5 \mathrm{eV}$ for the positive bubble. After passing the off axis positive bubble the streamer regains its unperturbed form.

\section{Mean free path of ionizing photons}

The results discussed here are sensitive to the mean free path for ionizing radiation, $\lambda_{\mathrm{P}}$, whose value determines the dimensions of the streamer and its speed. For example, streamer branching on a positive bubble ( $80 \mu \mathrm{m}$ radius) with $\lambda_{\mathrm{P}}=220 \mu \mathrm{m}$ is shown in figure 17 where the streamer width is commensurate with the bubble. For otherwise identical conditions, the streamer intersection is shown in figure 18 for $\lambda_{\mathrm{P}}=880 \mu \mathrm{m}$. The increase in mean free path results in a broader streamer making the bubble radius smaller in comparison. The end result is that branching does not occur. More details on the influence of $\lambda_{\mathrm{P}}$ on streamer-bubble dynamics are discussed in Part II [29].

$$
P / P_{0}=1.08
$$

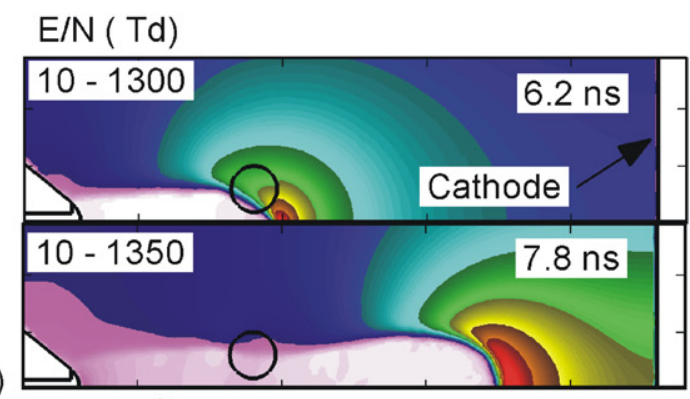

[e] $\left(\mathrm{cm}^{-3}(3 \mathrm{dec})\right)$

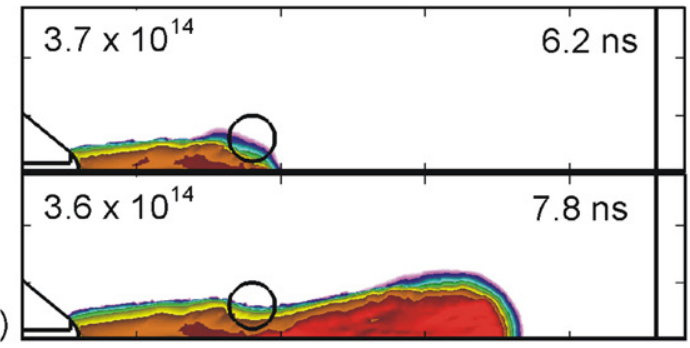

(b) $\mathrm{T}_{\mathrm{e}}(\mathrm{eV})$

(c)

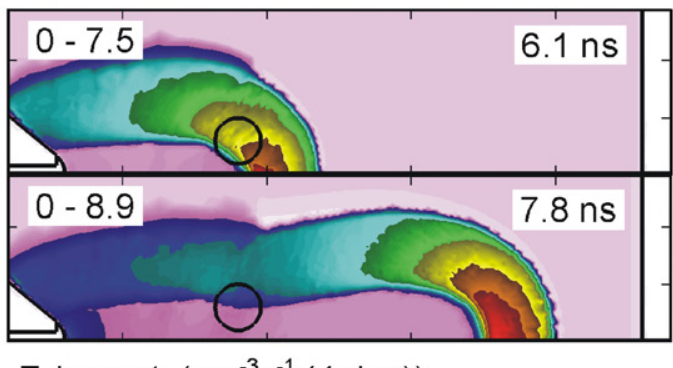

(d)

E-Impact $\left(\mathrm{cm}^{-3} \mathrm{~s}^{-1}(4 \mathrm{dec})\right)$

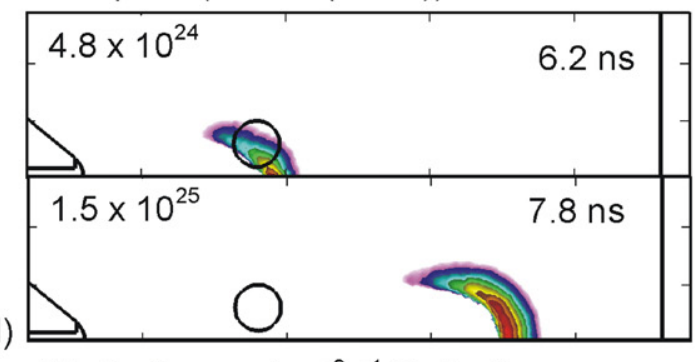

Photo Source $\left(\mathrm{cm}^{-3} \mathrm{~s}^{-1}(3 \mathrm{dec})\right)$

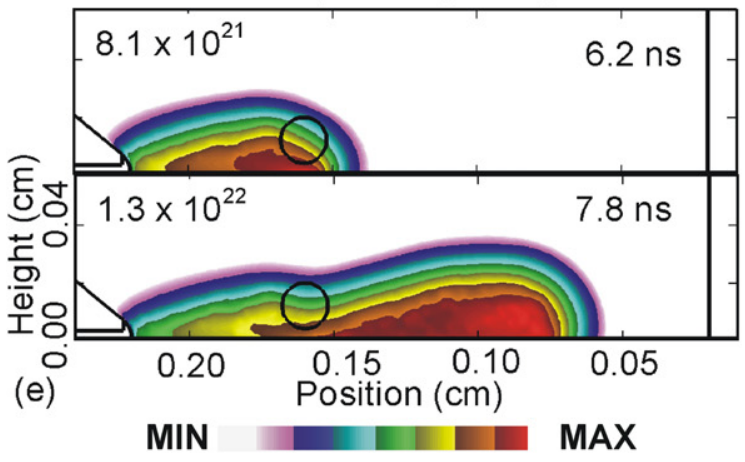

Figure 16. Plasma properties for a streamer approaching a positive bubble $\left(P / P_{0}=1.08\right)$ of $80 \mu \mathrm{m}$ radius centered $120 \mu \mathrm{m}$ off axis at 6.2 and 7.8 ns: $(a) E / N,(b)$ electron density, $(c) T_{\mathrm{e}},(d)$ electron impact ionization and $(e)$ photoionization sources. The streamer tends to avoid the bubble and squeezes to the axis. No branching occurs. The range of values or maximum value plotted are indicated in each frame. Log plots are indicated by the number of decades. 


$$
\begin{aligned}
& \lambda_{\mathrm{ph}}=220 \mu \mathrm{m} \\
& P / P_{0}=1.08
\end{aligned}
$$

Photo Source $\left(\mathrm{cm}^{-3} \mathrm{~s}^{-1}(4 \mathrm{dec})\right)$

(a)

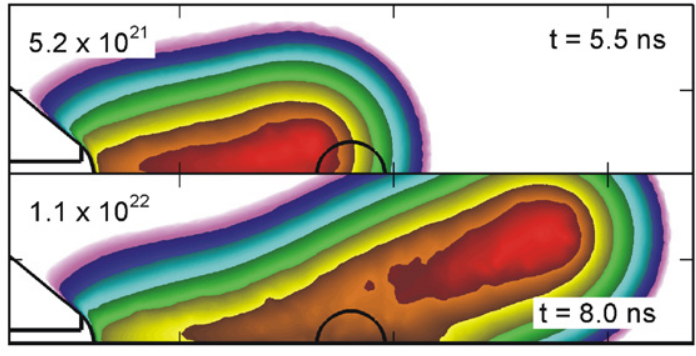

[e] $\left(\mathrm{cm}^{-3}(3 \mathrm{dec})\right)$

(b)

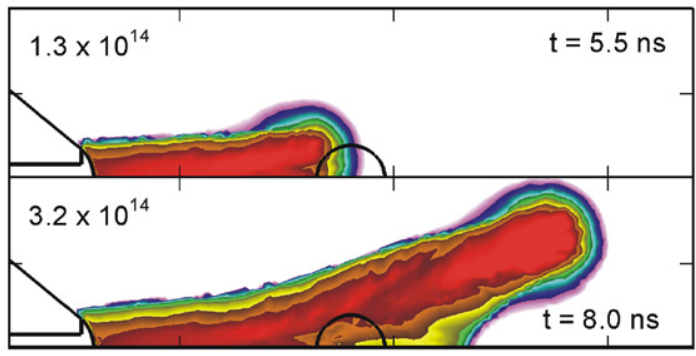

Positive Charge $\left(\mathrm{cm}^{-3}(2 \mathrm{dec})\right)$

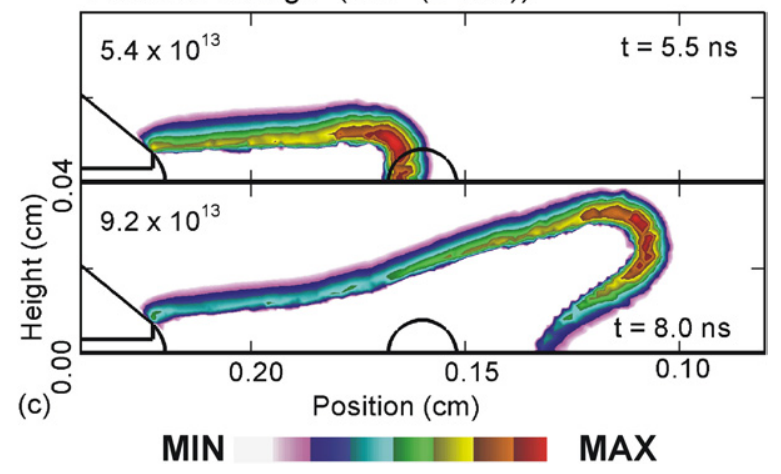

Figure 17. Plasma properties for a streamer approaching a positive bubble $\left(P / P_{0}=1.08\right)$ of $80 \mu \mathrm{m}$ radius at 5.5 and $8.0 \mathrm{~ns}$ for $\lambda_{\mathrm{P}}=220 \mu \mathrm{m}$ : (a) photoionization source, $(b)$ electron density and (c) positive space charge. With a short $\lambda_{\mathrm{P}}$ branching on the positive bubble occurs. The maximum value for log plots and number of decades are indicated in each frame.

\section{Concluding remarks}

Positive corona streamer dynamics in humid air intersecting with positive and negative bubbles, and solid particles on or near the streamer axis were computationally investigated. Both positive and negative bubbles can significantly perturb the stream dynamics, effects that depend on the size of the bubble and $P / P_{0}$. Bubbles smaller than the width of the streamer with $0.92<P / P_{0}<1.08$ (the range discussed here) have little effect on streamer dynamics as they are enveloped by the streamer. Bubbles comparable in size to the streamer do influence streamer dynamics. We found that streamers tend to branch when encountering a positive bubble on the streamer axis whereas a streamer accelerates into and penetrates a negative bubble on axis. Conversely, a streamer branches into a negative bubble off axis and is compressed into the axis when encountering an off axis positive bubble. Streamers encountering a solid bubble (a particle) may branch or initiate
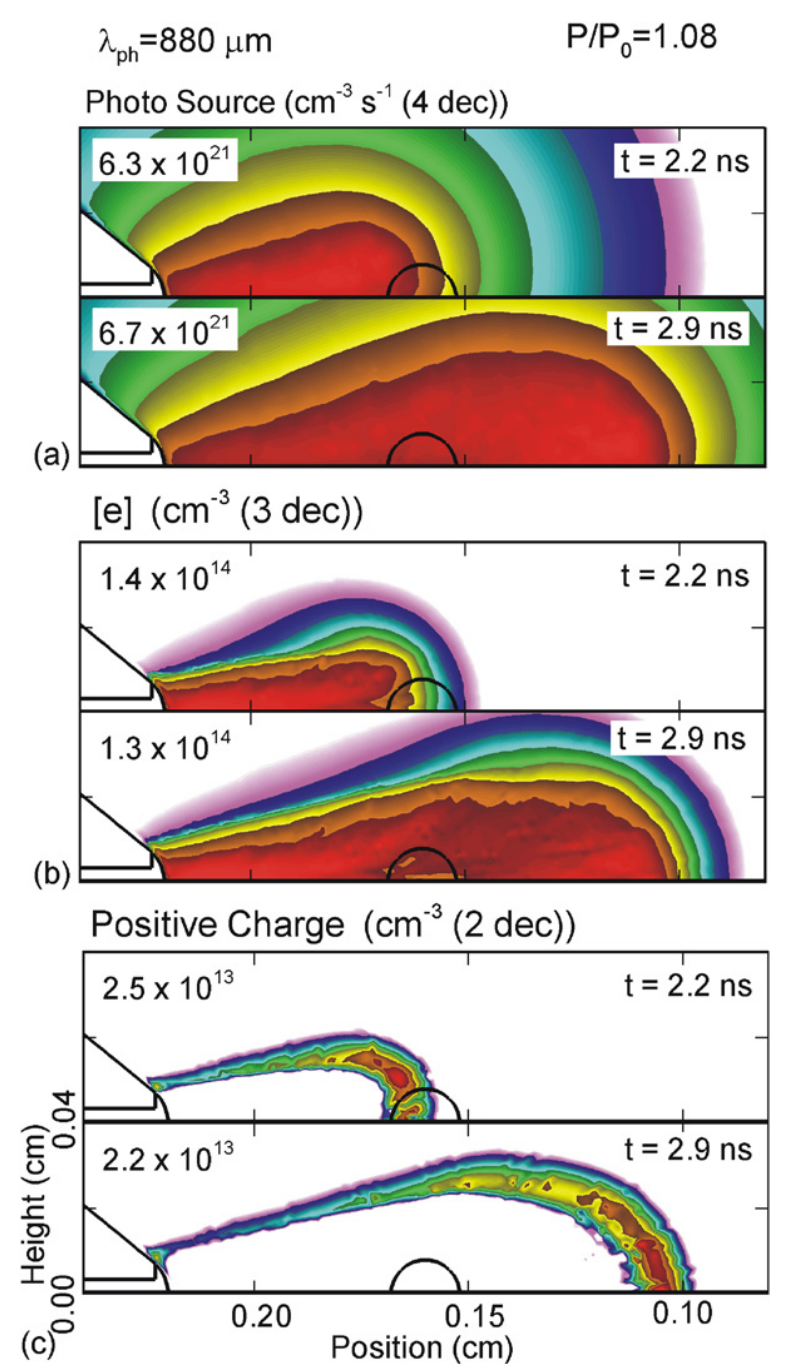

MIN

MAX

Figure 18. Plasma properties for a streamer approaching a positive bubble $\left(P / P_{0}=1.08\right)$ of $80 \mu \mathrm{m}$ radius at 2.2 and $2.9 \mathrm{~ns}$ for $\lambda_{\mathrm{P}}=880 \mu \mathrm{m}:(a)$ photoionization source, $(b)$ electron density and (c) positive space charge. With a long $\lambda_{\mathrm{P}}$, the streamer speed is higher, width is larger and the streamer envelopes the bubble without branching. The maximum value for $\log$ plots and number of decades are indicated in each frame.

new streamers, depending on the permittivity and size of the particle. Small solid particles with low dielectric constants are enveloped by the streamer plasma and may produce branching. Particles with high dielectric constant stall and reinitiate the streamer.

\section{References}

[1] Nasser E and Loeb L B 1963 J. Appl. Phys. 343340

[2] Bazelyan E M and Raizer Yu P 1998 Spark Discharge (Boca Raton, FL: CRC Press)

[3] Briels T M P, van Veldhuizen E M and Ebert U 2005 Trans. Plasma Sci. 33264

[4] Cummer S A, Jaugey N, Li J, Lyons W A, Nelson T E and Gerken E A 2006 Geophys. Res. Lett. 33 L04104

[5] Wang F, Liu J B, Sinibaldi J, Brophy C, Kuthi A, Jiang C, Ronney P and Gundersen M A 2005 Trans. Plasma Sci. 33844 
[6] Chang J S 2008 Plasma Sources Sci. Technol. 17045004

[7] van Veldhuizen E M and Rutgers W R 2002 J. Phys. D: Appl. Phys. 352169

[8] Tardiveau P, Marode E and Agneray A 2002 J. Phys. D: Appl. Phys. 352823

[9] Pancheshnyi S, Nudnova M and Starikovskii A 2005 Phys. Rev. E 71016407

[10] Babaeva N Yu and Naidis G V 1996 J. Phys. D: Appl. Phys. 292423

[11] Tardiveau P and Marode E 2003 J. Phys. D: Appl. Phys. 361204

[12] Oladiran E O 1981 J. Pure Appl. Geophys. 119966

[13] Oladiran E O 1982 J. Pure Appl. Geophys. 120673

[14] Timatkov V V, Pietsch G J, Saveliev A B, Sokolova M V and Temnikov A G 2005 J. Phys. D: Appl. Phys. 38877

[15] Sigmond R S, Sigmond T, Rolfseng L, Bohman A F, Stormo F T and Hvidsten L 2004 Trans. Plasma Sci. 321812

[16] Briels T M P, Kos J, van Veldhuizen E M and Ebert U 2006 J. Phys. D: Appl. Phys. 395201

[17] Winands G J J, Liu Z, Pemen A J M, van Heesch E J M and Yan K 2008 J. Phys. D: Appl. Phys. 41234001

[18] Luque A, Ebert U and Hundsdorfer W 2008 Phys. Rev. Lett. 101075005

[19] Nijdam S, Moerdam J S, Briels T M P, van Veldhuizen E M and Ebert U 2008 Appl. Phys. Lett. 92101502

[20] Naidis G V 1996 J. Phys. D: Appl. Phys. 29779

[21] Arrayas M, Ebert U and Hundsdorfer W 2002 Phys. Rev. Lett. 88174502

[22] Rocco A, Ebert U and Hundsdorfer W 2002 Phys. Rev. E 66035102
[23] Meulenbroek B, Rocco A and Ebert U 2004 Phys. Rev. E 69067402

[24] Ebert U, Montijn C, Briels T M P, Hundsdorfer W, Meulenbroek B, Rocco A and van Veldhuizen E M 2006 Plasma Sources Sci. Technol. $15 \mathrm{~S} 118$

[25] Ebert U and Sentman D D 2008 J. Phys. D: Appl. Phys. 41230301

[26] Starikovskaia S M, Kukaev E N, Kuksin A Yu, Nudnova M M and Starikovskii A Yu 2004 Combust. Flame 139177

[27] Masuda S and Hosokawa S 1995 Handbook of Electrostatic Processes ed J S Chang et al (New York: Dekker)

[28] Babaeva N Yu, Bhoj A N and Kushner M J 2006 Plasma Sources Sci. Technol. 15591

[29] Babaeva N Yu and Kushner M J 2009 Plasma Sources Sci. Technol. 18035010

[30] Naidis G V 2006 Plasma Sources Sci Technol. 15253

[31] Bourdon A, Pasko V P, Liu N Y, Célestin S, Ségur P and Marode E 2007 Plasma Sources Sci. Technol. 16656

[32] Liu N, Celestin S, Bourdon A, Pasko V P, Ségur P and Marode E 2007 Appl. Phys. Lett. 91211501

[33] Luque A, Ebert U, Montijn C and Hundsdorfer W 2007 Appl. Phys. Lett. 901501

[34] Nudnova M M and Starikovskii A Yu 2008 J. Phys. D: Appl. Phys. 41234003

[35] Cheng D K 1992 Field and Wave Electromagnetics (Reading, MA: Addison-Wesley)

[36] Babaeva N Yu, Lee J K and Kim H C 2004 Plasma Sources Sci. Technol. 13127 